\title{
Simultaneous Localization and Calibration for Cooperative Radio Navigation
}

\author{
Robert Pöhlmann, Siwei Zhang, Emanuel Staudinger, Armin Dammann, and Peter A. Hoeher, Fellow, IEEE
}

\begin{abstract}
Cooperative radio localization and navigation systems can be used in scenarios where the reception of global navigation satellite system (GNSS) signals is not possible or impaired. While the benefit of cooperation has been highlighted by many papers, calibration is not widely considered, but equally important in practice. Utilizing the signal propagation time requires group delay or ranging bias calibration and estimating the direction-of-arrival $(\mathrm{DoA})$ requires antenna response calibration. Often, calibration parameters are determined only once before operation. However, the calibration parameters are influenced by e.g. changing temperatures of radio frequency (RF) components or changing surroundings of antennas. To cope with that, we derive a cooperative simultaneous localization and calibration (SLAC) algorithm based on Bayesian filtering, which estimates antenna responses and ranging biases simultaneously with positions and orientations. By simulations, we show that the calibration parameters can be estimated during operation without additional sensors. We further proof practical applicability of SLAC by evaluating measurement data from robotic rovers. With SLAC, both ranging and DoA estimation performance is improved, resulting in better position and orientation estimation accuracy. SLAC is thus able to provide reliable calibration and to mitigate model mismatch. Finally, we discuss open research questions and possible extensions of SLAC.
\end{abstract}

Index Terms-Antenna arrays, antenna response, array signal processing, direction-of-arrival, multi-mode antenna, ranging

\section{INTRODUCTION}

$\mathbf{M}$ ANY applications require localization with defined accuracy and reliability. Outdoors, global navigation satellite systems (GNSSs) often fulfill the requirements. However, for indoor localization [1] and Internet of Things (IoT) [2], [3], GNSS signal reception is impaired. Furthermore, systems complementary to GNSS are considered for safetycritical applications like smart vehicles and autonomous driving [4], [5]. Localization is also a key component of 5G and $6 \mathrm{G}$ cellular networks [6]-[9]. A scenario where neither GNSS nor other infrastructure is available, is autonomous planetary exploration by a robotic swarm or multi-agent system [10], [11]. Navigation of a robotic multi-agent system is particularly examined in this paper, however the presented method is general.

Robert Pöhlmann, Siwei Zhang, Emanuel Staudinger and Armin Damman are with the Institute of Communications and Navigation, German Aerospace Center (DLR), Wessling 82234, Germany (e-mail: \{Robert.Poehlmann, Siwei.Zhang, Emanuel.Staudinger, Armin.Dammann\} @dlr.de).

Peter A. Hoeher is with the University of Kiel, Faculty of Engineering, Kiel 24143, Germany (e-mail: ph@tf.uni-kiel.de).

Manuscript received Month xx, xxxx; revised Month xx, xxxx.

This works has partly been funded by the Helmholtz Association Project "Autonomous Robotic Networks to Help Modern Societies (ARCHES)" under Grant ZT-0033.
Looking at cooperative localization on an abstract level, typically there are static nodes in the network with known positions, which are called anchors. Besides, there are mobile nodes with unknown position and orientation, which we call agents. In many cases the orientations of the agents is also important, e.g. to control a robot or to predict the motion of a vehicle. Often position and orientation estimation are considered jointly [8], [9], [12]. By the term localization, we thus consider both, position and orientation estimation. Joint position and orientation estimation with radio signals is possible by exploiting distance information, e.g. using a twoway ranging (TWR) protocol, and direction information using multiport antennas, e.g. phased arrays, co-located antennas or multi-mode antennas (MMAs) [13].

Radio localization methods can be categorized into noncooperative and cooperative. For non-cooperative localization, only agents and anchors communicate, whereas for cooperative localization, agents also exchange signals among each other. Theoretical investigation of fundamental limits has shown that cooperative is superior to non-cooperative localization [14]-[17]. The theoretical results have been confirmed by measurements [18]. Many algorithms for cooperative localization have been proposed, see [19], [20] and the references therein.

An aspect which we deem to be equally important as cooperation for a practical system, but which has received much less attention in the community so far, is calibration. An improperly calibrated system suffers from biased estimation and thus impaired performance. To highlight the importance of calibration for cooperative localization, we take a look at the architecture of GNSS for comparison. For GNSS, highgrade hardware on the space segment and a dedicated control segment allow the use of low-cost commercial off-the-shelf (COTS) hardware on the user segment. For cooperative localization, in contrary, an agent relies to a large extent on signals transmitted by its neighbors. There may still be anchors, but usually the infrastructure is sparse. Furthermore, for many applications, the radio frequency (RF) hardware will consist of low-cost COTS components. For a propagation time based system, e.g. using the signal time-of-flight $(\mathrm{ToF})$ or time-ofarrival (ToA), group delays in the transmit and receive chains and the antenna of the node transceivers need to be calibrated. The calibration is often done in a once for all fashion before operation, by connecting the node transceivers to a calibrated reference to measure the group delays. However, transceiver internal group delays can change over time, for instance due to temperature variations, which results in a ranging bias. Low-cost COTS hardware is especially prone to group 
delay variations. For direction-of-arrival (DoA) or directionof-departure (DoD) estimation, accurate knowledge of the antenna response of the receiving multiport antenna is required. As the surrounding structure of antenna influences its response, the entire device needs to fit into a measurement chamber to obtain the installed antenna response. Since this is difficult and costly when the antenna is installed on a large vehicle, usually the antenna alone is measured in a near-field measurement chamber. However, the installed antenna response generally deviates from the stand-alone antenna response, which leads to a model mismatch and biased DoA estimates [21].

Even if the antenna is calibrated in its final installation position, e.g. on a robot, calibrating the antenna response and group delay only once before operation is conceptually problematic for the case where the surrounding of the antenna during operation changes. As an example consider a robot with a manipulator arm, allowing it to grab and carry payload boxes [22]. As the manipulator arm and a potential payload box are in close vicinity to the antenna, they influence the antenna response. Regarding planetary exploration and space missions, there is the impact of launch and landing and the harsh space environment, e.g. dust, radiation and large temperature differences. Thus, there is a need to verify the nominal condition of the system, and re-calibration must be done in-situ during the mission.

In contrast to calibrating once for all, calibration parameters can in general also be determined during operation. For simultaneous localization and mapping (SLAM), calibration is often considered as integral part, see e.g. the conceptual papers [23], [24]. Examples include SLAM with robot odometry calibration [25] and a SLAM approach to microphone array calibration [26]. In [27], SLAM is applied to electromagnetic localization of instruments in patient's body during surgery and calibration of electromagnetic distortions. In [28], a method to calibrate ranging biases in a sensor network is proposed, however there is no cooperation between the sensors. In the recent paper [29], SLAM is applied to calibrate a uniform linear array (ULA) of an automotive radar, using targets of opportunity. The approach is limited to amplitude and phase calibration of the individual ports, other antenna response deviations cannot be corrected. SLAM has recently gained attention for radio localization in the context of emerging millimeter wave (mmWave) communications for $5 \mathrm{G}$ and $6 \mathrm{G}$ cellular networks. Due to the high temporal and spatial resolution of mmWave systems, parameters of each propagation path can be estimated, which even allows to utilize multipath propagation for localization [9]. With the principle of SLAM, the position and orientation of a node and a map of its environment are determined simultaneously [30]-[32]. Thus, accurate position and orientation estimation is possible even in pure non-line-ofsight (NLoS) conditions. When the main focus is calibration instead of mapping, often the term simultaneous localization and calibration (SLAC) is used. Examples can be found in [33], where radio-frequency identification (RFID) tags are localized while RFID tag positions are calibrated, and [34], where indoor localization with fingerprinting is performed, while calibrating sensor and walking model.

However, SLAC has not been widely considered for radio localization in cooperative networks or as part of SLAM using radio signals. Especially, there is no approach to estimate the position and orientation and simultaneously calibrate the antenna response of an arbitrary multiport antenna.

The aim of this paper is to introduce cooperative SLAC, where key parameters required for cooperative localization, namely antenna responses and ranging biases, are estimated during operation. This constitutes a paradigm shift towards practical considerations and model mismatch mitigation in the algorithm design. The main contributions of this paper are as follows:

- We present a novel method to simultaneously calibrate an antenna and determine its position and orientation in a cooperative network. In contrast to [29], the method can be applied to arbitrary antenna types, e.g. phased arrays, co-located antennas or MMAs, and allows a full calibration of the antenna, including gain-phase offsets, mutual coupling and nonlinear antenna response deviations.

- We show how cooperative SLAC can be implemented as a Bayesian filtering algorithm. For that, we define the signal model and state space for the unknown position, orientation, ranging bias and antenna response of the nodes. The antenna response is represented in a parameterized fashion by wavefield modeling and manifold separation. We present suitable motion and measurement models and consider prior knowledge of the calibration parameters.

- We perform extensive simulations for random scenarios and antenna responses, where we show that ranging biases and antenna responses can be estimated simultaneously to localization without additional sensors. For realistic ranging biases and antenna response deviations, cooperative SLAC outperforms cooperative localization and shows considerably lower position and orientation errors.

- As SLAC is motivated by practical considerations, we also evaluate the proposed algorithm with measurement data from robotic rovers carrying software-defined radios (SDRs). One of the rovers features a four-port MMA to enable DoA estimation. By SLAC, both the ranging performance and the DoA estimation performance are improved, which indicates correct calibration. After all, position and orientation estimation performance is improved as well, demonstrating that SLAC performs well in a practical scenario.

- Finally, we discuss open research questions regarding cooperative SLAC, which are beyond the scope of this paper. Parameter observability deserves a theoretical analysis and the performance in multipath conditions should be investigated. The generic architecture of SLAC allows the inclusion of other sensors in the calibration and the implementation in a distributed fashion.

The paper is organized as follows. In Section II, we introduce the signal model based on wavefield modeling and manifold separation. The state space as well as the transition and measurement models are defined in Section III. We then derive the SLAC algorithm in Section IV as a Bayesian filter. The algorithm is evaluated by extensive simulations presented 


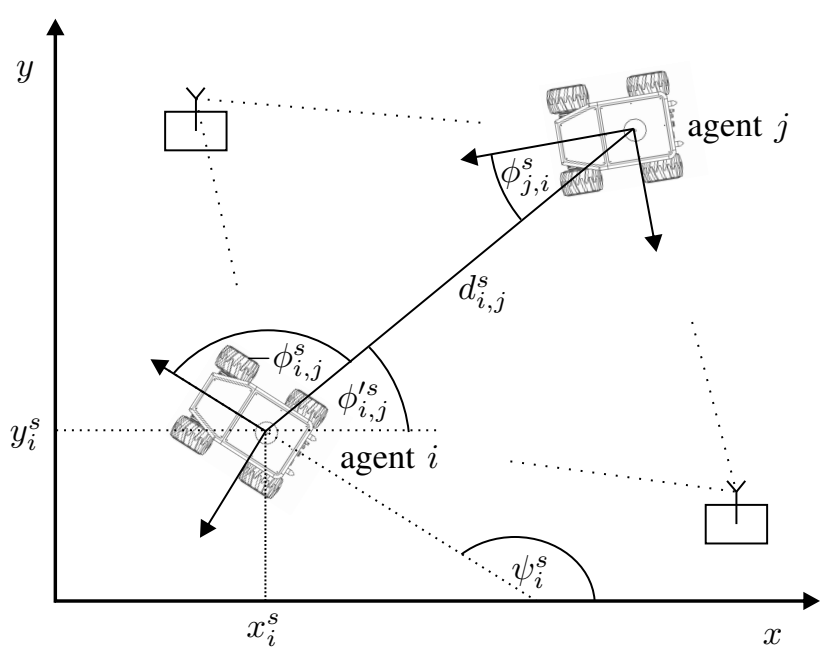

Fig. 1. Network of four nodes: Two anchors and two agents $i$ and $j$, the distance $d_{i, j}^{s}$ in between the agents, the DoAs $\phi_{i, j}^{s}$ and $\phi_{j, i}^{s}$ and the position $\boldsymbol{p}_{i}^{s}=\left[\begin{array}{ll}x_{i}^{s} & y_{i}^{s}\end{array}\right]^{T}$ and orientation $\psi_{i}^{s}$ of agent $i$.

in Section V. In Section VI we introduce the measurement setup and further evaluate the algorithm with measurement data. In Section VII we discuss parameter observability, multipath robustness, the inclusion of other sensors and distributed implementation. Section VIII concludes the paper.

Notation: Vectors are written in bold lowercase letters and matrices in bold capital letters. $(\cdot)^{T}$ and $(\cdot)^{H}$ stand for vector or matrix transpose and conjugate transpose, respectively. $\mathbf{1}_{N}$ and $\mathbf{0}_{N}$ are column vectors of ones and zeros of length $N$, $\mathbb{I}_{N}$ is an $N \times N$ identity matrix. $\|\boldsymbol{a}\|$ is the Euclidean norm. Square brackets refer to an element in a vector $[\boldsymbol{a}]_{b}$ or a matrix $[\boldsymbol{A}]_{b, c} \cdot[\boldsymbol{A}]_{\boldsymbol{b}, \boldsymbol{c}}$ denotes a sub-matrix of the entries indexed by vectors $\boldsymbol{b}$ and $\boldsymbol{c} . \boldsymbol{A}=\operatorname{diag}\{\boldsymbol{a}\}$ creates a diagonal matrix and $\boldsymbol{a}=\operatorname{vec}\{\boldsymbol{A}\}$ vectorizes $\boldsymbol{A}$ by stacking its columns. $\operatorname{Re}\{\cdot\}$, $\operatorname{Im}\{$.$\} refer to real and imaginary part and \lfloor a\rfloor$ is the floor function. $|a|$ denotes the absolute value of the scalar $a$ and $|\mathbb{A}|$ the cardinality of the set $\mathbb{A}$. $\arctan 2(y, x)$ is the four-quadrant inverse tangent.

\section{Signal Model}

Fig. 1 shows an exemplary drawing of a network of four nodes, consisting of two anchors and two agents. The distance between the two nodes $i$ and $j$ for snapshot $s$ is given by

$$
d_{i, j}^{s}=\left\|\boldsymbol{p}_{j}^{s}-\boldsymbol{p}_{i}^{s}\right\|=\frac{c\left(\tau_{i, j}^{s}-\bar{\tau}_{i, j}^{s}\right)}{2}+\delta_{i}^{s}+\delta_{j}^{s},
$$

with the position of node $i, \boldsymbol{p}_{i}^{s}=\left[\begin{array}{ll}x_{i}^{s} & y_{i}^{s}\end{array}\right]^{T}$, the position of node $j$ defined analogously, and the speed of light $c$. Assuming a TWR protocol, $\bar{\tau}_{i, j}^{s}$ is the transmit time of the forward signal and $\tau_{i, j}^{s}$ is the ToA of the backward signal, both at node $i$, and $\delta_{i}^{s}$ and $\delta_{j}^{s}$ are the ranging biases of nodes $i$ and $j$, respectively. We assume that the processing time between forward and backward transmission at node $j$ is already compensated and very short, such that the impact of the relative frequency offset of the two clocks can be neglected. Otherwise, the clock behavior must be taken into account with an appropriate clock model [35]. The ranging bias is caused by varying group delays in the transmitter and receiver chains, for instance due to temperature variations of the RF components. The signal DoA measured in the body frame of node $i$ is defined as

$$
\phi_{i, j}^{s}=\phi_{i, j}^{s \prime}-\psi_{i}^{s}=\arctan 2\left(y_{j}^{s}-y_{i}^{s}, x_{j}^{s}-x_{i}^{s}\right)-\psi_{i}^{s},
$$

where $\psi_{i}^{s}$ is the orientation of node $i$.

A signal sent by node $j$, received by node $i$, sampled at rate $B$ and transformed to discrete frequency domain can be written as

$$
\boldsymbol{r}_{i, j}^{s}(n)=\boldsymbol{a}_{i}\left(\phi_{i, j}^{s}\right) s\left(n, \tau_{i, j}^{s}\right) \alpha_{i, j}^{s} e^{\mathrm{j} \varphi_{i, j}^{s}}+\boldsymbol{w}_{i, j}^{s}(n),
$$

where $n \in\{1, \ldots, N\}$ is the discrete Fourier transform (DFT) bin index, $\boldsymbol{a}_{i}\left(\phi_{i, j}^{s}\right)$ is the antenna response of node $i$,

$$
s(n, \tau)=s(n) e^{-\mathrm{j} 2 \pi \tau B \frac{n-1}{N}}
$$

is a delayed version of the transmitted signal $s(n)$ in discrete frequency domain and $\alpha_{i, j}^{s}$ and $\varphi_{i, j}^{s}$ are amplitude and phase of the signal. The signal model (3) does not consider multipath propagation explicitly, see Section VII-B for a discussion. We assume that the signal bandwidth is small relative to the carrier frequency [36], and consider internal receiver noise $\boldsymbol{w}_{\boldsymbol{r}}^{s}(n) \sim \mathcal{C N}\left(0, \sigma_{\boldsymbol{r}_{i, j}^{s}}^{2} \mathbb{I}_{M_{i}}\right)$ for $M_{i}$ antenna ports as independent and identically distributed (i.i.d.) white circular symmetric Gaussian noise with variance $\sigma_{\boldsymbol{r}_{i, j}^{s}}^{2}$. The vectors

$$
\begin{gathered}
\boldsymbol{r}_{i, j}^{s}=\left[\begin{array}{lll}
\left(\boldsymbol{r}_{i, j}^{s}(1)\right)^{T} & \ldots & \left(\boldsymbol{r}_{i, j}^{s}(N)\right)^{T}
\end{array}\right]^{T}, \\
\boldsymbol{s}\left(\tau_{i, j}^{s}\right)=\left[\begin{array}{lll}
s\left(1, \tau_{i, j}^{s}\right) & \ldots & s\left(N, \tau_{i, j}^{s}\right)
\end{array}\right]^{T}
\end{gathered}
$$

are defined for later usage.

Two different antenna types are considered for the nodes. The nodes with singleport antenna, $M_{i}=1$, are assumed to be equipped with half-wavelength dipoles, such that their antenna response

$$
a_{i}(\phi)=10^{\frac{2.15 \mathrm{dBi}}{20}}
$$

is independent of the DoA $\phi$. For the nodes with multiport antenna, i.e. antenna ports $m=\left\{1, \ldots, M_{i}\right\}$, the gain pattern $g_{i, m}(\phi)$ and phase pattern $\Phi_{i, m}(\phi)$ are considered [36], such that the antenna response for port $m$ of node $i$ is

$$
a_{i, m}(\phi)=\sqrt{g_{i, m}(\phi)} e^{\mathrm{j} \Phi_{i, m}(\phi)},
$$

and the antenna response vector is:

$$
\boldsymbol{a}_{i}(\phi)=\left[\begin{array}{lll}
a_{1}(\phi) & \ldots & a_{M_{i}}(\phi)
\end{array}\right]^{T} .
$$

While the gain pattern $g_{i, m}(\phi)$ and phase pattern $\Phi_{i, m}(\phi)$ of an ideal antenna array can be described in closed-form [37], finding a closed-form description of e.g. co-located antennas or MMAs is not straightforward. Furthermore, real-world antenna arrays in general do not behave according to ideal models [21]. We thus consider a generic antenna model by using wavefield modeling and manifold separation [38], [39], which allows to represent arbitrary antenna responses. Like that, it is possible to apply the same signal model and state space to phased arrays, co-located antennas, MMAs and any other type of multiport antennas. The antenna response vector (9) can be decomposed into a product of the sampling matrix $\boldsymbol{G}_{i} \in \mathbb{C}^{M_{i} \times U}$, which describes the antenna characteristics, 
and a predefined basis vector $\boldsymbol{b}(\phi) \in \mathbb{C}^{U}$, which is a function of the DoA:

$$
\boldsymbol{a}_{i}(\phi)=\boldsymbol{G}_{i} \boldsymbol{b}(\phi) .
$$

The antenna response needs to be square integrable and the basis functions must be orthonormal on the manifold $\phi \in$ $[-\pi, \pi)[38],[39]$. As basis we use the Fourier functions:

$$
\boldsymbol{b}(\phi)=\frac{1}{\sqrt{2 \pi}} e^{\mathrm{j} \phi u_{\phi}}, u_{\phi}=\left\lfloor-\frac{U-1}{2}\right\rfloor, \ldots, 0, \ldots,\left\lfloor\frac{U-1}{2}\right\rfloor .
$$

The order $U$ of the basis functions can be determined based on the electrical size of the antenna [38]. It is possible to apply wavefield modeling and manifold separation also to joint estimation of azimuth and elevation using spherical harmonics or 2D Fourier functions as basis [13], [38], [39]. However, the scope of this paper is $2 \mathrm{D}$ position and orientation estimation, therefore we only consider azimuth.

During the design process of antennas, usually electromagnetic (EM) simulations are performed. Once an antenna is manufactured, it is often measured in a near-field measurement chamber to ensure it meets the specifications. Whether from simulation or from a measurement, often spatial samples of the antenna response are available. They can be seen as a sampled version of (9) and are proportional to the electric field strength. Omitting the node index $i$ for notational convenience, the spatial samples with index $q \in\{1, \ldots, Q\}$ for antenna ports $m \in\{1, \ldots, M\}$ obtained at DoAs $\phi_{q}$ can be written as

$$
\begin{gathered}
\boldsymbol{e}_{q}=\left[\begin{array}{lll}
e_{q, 1} & \ldots & e_{q, M}
\end{array}\right]^{T}, \\
\boldsymbol{E}^{0}=\left[\begin{array}{lll}
\boldsymbol{e}_{1} & \ldots & \boldsymbol{e}_{Q}
\end{array}\right] .
\end{gathered}
$$

The spatial sampling grid must be dense enough, such that the spatial sample-rate criterion according to the Nyquist theorem is fulfilled. A prior sampling matrix

$$
\hat{\boldsymbol{G}}_{i}^{0}=\boldsymbol{E}_{i}^{0} \boldsymbol{B}^{H}\left(\boldsymbol{B} \boldsymbol{B}^{H}\right)^{-1},
$$

can then be obtained by the least squares method, with $\boldsymbol{B}=$ $\left[\boldsymbol{b}\left(\phi_{1}\right), \ldots, \boldsymbol{b}\left(\phi_{Q}\right)\right]$. However, EM simulation does not account for manufacturing imperfections. Measuring the antenna alone in a near-field measurement chamber neglects the influence of the surrounding structure on the antenna response. The prior sampling matrix $\hat{G}_{i}^{0}$ from (13) will thus deviate from the true sampling matrix $\boldsymbol{G}_{i}$, causing a model mismatch and impaired DoA estimation performance. The SLAC algorithm proposed in this paper uses $\hat{G}_{i}^{0}$ as prior information on the antenna response, see (40), which is gradually refined over time.

\section{State Space}

\section{A. Variables and Measurements}

The network consists of $|\mathbb{D}|$ nodes listed in the node set $\mathbb{D}$. There are two types of nodes: Anchors are static nodes with known position and orientation and agents are mobile nodes, which shall be localized. Agents are referred to by the agent set $\mathbb{A} \subset \mathbb{D}$. The node state vector of an agent $i \in \mathbb{D}$ is composed of the node kinematic states $\boldsymbol{x}_{i, \text { loc }}^{s}$ and the node calibration parameter states $\boldsymbol{x}_{i, \text { cal }}^{s}$ :

$$
\boldsymbol{x}_{i}^{s}=\left[\begin{array}{ll}
\left(\boldsymbol{x}_{i, \mathrm{loc}}^{s}\right)^{T} & \left(\boldsymbol{x}_{i, \mathrm{cal}}^{s}\right)^{T}
\end{array}\right]^{T} .
$$

The node state vector of an anchor $i \in \mathbb{D}$ consists only of the node calibration parameter states: $\boldsymbol{x}_{i}^{s}=\boldsymbol{x}_{i, \text { cal }}^{s}$. Node kinematic states are defined as

$$
\boldsymbol{x}_{i, \text { loc }}^{s}=\left[\begin{array}{llll}
\left(\boldsymbol{p}_{i}^{s}\right)^{T} & v_{i}^{s} & \psi_{i}^{s} & \omega_{i}^{s}
\end{array}\right]^{T},
$$

with position $\boldsymbol{p}_{i}^{s}$, linear velocity $v_{i}^{s}$, orientation $\psi_{i}^{s}$ and angular velocity $\omega_{i}^{s}$. Node calibration states are given by

$$
\boldsymbol{x}_{i, \text { cal }}^{s}=\left[\begin{array}{ll}
\delta_{i}^{s} & \left(\boldsymbol{g}_{i}^{s}\right)^{T}
\end{array}\right]^{T},
$$

with the ranging bias $\delta_{i}^{s}$ and the sampling matrix entries represented in vectorized form,

$$
\boldsymbol{g}_{i}^{s}=\left[\begin{array}{l}
\operatorname{Re}\left\{\operatorname{vec}\left\{\boldsymbol{G}_{i}^{s}\right\}\right\} \\
\operatorname{Im}\left\{\operatorname{vec}\left\{\boldsymbol{G}_{i}^{s}\right\}\right\}
\end{array}\right],
$$

which are only present for multiport nodes $M_{i}>1$. For localization-only, $\boldsymbol{x}_{i \text {,cal }}^{s}$ is not part of the node state vector. The state vector for the entire network is formed by stacking the node states:

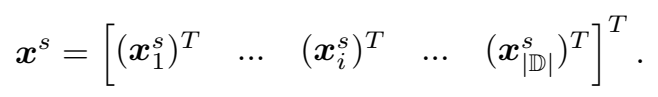

The vectors $\boldsymbol{\iota}_{i}, \boldsymbol{\iota}_{i, \text { loc }}, \boldsymbol{\iota}_{i, \text { cal }}$ contain the indices of the entries of $\boldsymbol{x}_{i}^{s}, \boldsymbol{x}_{i, \text { loc }}^{s}, \boldsymbol{x}_{i, \text { cal }}^{s}$ in the state vector $\boldsymbol{x}^{s}$, respectively. For snapshot $s$, node $i$ receives signals $\boldsymbol{r}_{i, j}^{s}$, see (3) and (5), from neighboring nodes $j \in \mathbb{L}_{i}^{s}$, which are listed in the neighbor set $\mathbb{L}_{i}^{s} \subseteq \mathbb{D} \backslash\{i\}$. If e.g. a neighboring node is too far away from node $i$ or the signal is blocked, it is not a member of $\mathbb{L}_{i}^{s}$. As TWR requires bidirectional links, the corresponding reverse signal $\boldsymbol{r}_{j, i}^{s}$ is always present. The received signals from all nodes in the network are collected in the measurement vector:

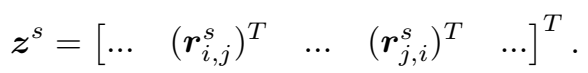

This corresponds to a centralized estimation approach, see the discussion in Section VII-D.

\section{B. State Transition}

For the kinematic states (15) of node $i$, the state transition from the previous snapshot $s^{-}=s-1$ to the current snapshot $s$ is described by the motion model

$$
\boldsymbol{x}_{i, \text { loc }}^{s}=\boldsymbol{f}\left(\boldsymbol{x}_{i, \text { loc }}^{s^{-}}\right)+\boldsymbol{w}_{\boldsymbol{x}_{i, \text { loc }}}^{s}
$$

with process noise $\boldsymbol{w}_{\boldsymbol{x}_{i, \text { loc }}}^{s}$. We use a constant turn model with polar velocity, which has been suggested in [40], [41] for aircraft tracking. Using trigonometric identities, it can be shown that the transition equations are equivalent to the motion model proposed in [42] for robotic rovers. For time interval $T$ between snapshots, the transition function is

$$
\boldsymbol{f}\left(\boldsymbol{x}_{i, \text { loc }}^{s^{-}}\right)=\left[\begin{array}{c}
x_{i}^{s^{-}}-\frac{v_{i}^{s^{-}}}{\omega_{i}^{s^{-}}} \\
\sin \left(\psi_{i}^{s^{-}}\right)+\frac{v_{i}^{s^{-}}}{\omega_{i}^{s^{-}}} \\
y_{i}^{s^{-}}+\frac{v_{i}^{s^{-}}}{\omega_{i}^{s^{-}}} \sin \left(\psi_{i}^{s^{-}}+\omega_{i}^{s^{-}} T\right) \\
\left.v_{i}^{s^{-}}\right)-\frac{v_{i}^{s^{-}}}{\omega_{i}^{s^{-}}} \cos \left(\psi_{i}^{s^{-}}+\omega_{i}^{s^{-}} T\right) \\
\psi_{i}^{s^{-}}+T \omega_{i}^{s^{-}} \\
\omega_{i}^{s^{-}}
\end{array}\right]
$$

and the process noise is Gaussian,

$$
\boldsymbol{w}_{\boldsymbol{x}_{i, \text { loc }}}^{s} \sim \mathcal{N}\left(\mathbf{0}_{5}, \boldsymbol{\Sigma}_{\boldsymbol{x}_{i, \text { loc }}}^{s}\right),
$$


with covariance matrix

$$
\boldsymbol{\Sigma}_{\boldsymbol{x}_{i, \mathrm{loc}}}^{s}=\left[\begin{array}{ccccc}
0 & 0 & 0 & 0 & 0 \\
0 & 0 & 0 & 0 & 0 \\
0 & 0 & T \sigma_{\dot{v}}^{2} & 0 & 0 \\
0 & 0 & 0 & \frac{1}{3} T^{3} \sigma_{\dot{w}}^{2} & \frac{1}{2} T^{2} \sigma_{\dot{w}}^{2} \\
0 & 0 & 0 & \frac{1}{2} T^{2} \sigma_{\dot{w}}^{2} & T \sigma_{\dot{w}}^{2}
\end{array}\right] .
$$

The process noise parameters $\sigma_{\dot{v}}^{2}$ and $\sigma_{\dot{w}}^{2}$ reflect changes in linear velocity and angular velocity, respectively. The calibration parameters $\boldsymbol{x}_{i, \text { cal }}^{s}$ are assumed to be constant over time with zero process noise.

\section{Measurement Model}

Many cooperative localization algorithms operate on extracted distance and/or direction information, which is referred to as two step approach. For cooperative SLAC, this is not possible. In order to calibrate a multiport antenna, the antenna response information must be visible to the algorithm. This is not the case for the two step approach, where only DoA and ToA estimates are available. For that reason, we directly consider the log-likelihood functions of the received signals. Compared to a two step approach, this one step approach is also beneficial in the case of multipath propagation, which we discuss in Section VII-B.

1) Singleport Antenna: For nodes with a singleport antenna, $M_{i}=1$, the log-likelihood function of the received signal (3) and (5) is given by:

$$
\begin{aligned}
& L_{i, j}^{s}\left(\tau_{i, j}^{s}, \alpha_{i, j}^{s}, \varphi_{i, j}^{s}\right)=-N \ln \left(\pi \sigma_{\boldsymbol{r}_{i, j}^{s}}^{2}\right) \\
& \quad-\frac{1}{\sigma_{\boldsymbol{r}_{i, j}^{s}}^{2}} \sum_{n=1}^{N}\left|r_{i, j}^{s}(n)-s\left(n, \tau_{i, j}^{s}\right) \alpha_{i, j}^{s} e^{\mathrm{j} \varphi_{i, j}^{s}}\right|^{2} .
\end{aligned}
$$

Plugging the minimum of (24) with respect to (w.r.t.) the unknown signal amplitude and phase back into (24) and neglecting the constant term, we obtain the concentrated loglikelihood function:

$$
\tilde{L}_{i, j}^{s}\left(\tau_{i, j}^{s}\right)=\frac{1}{\sigma_{\boldsymbol{r}_{i, j}^{s}}^{2}} \frac{\left|\boldsymbol{s}\left(\tau_{i, j}^{s}\right)^{H} \boldsymbol{r}_{i, j}^{s}\right|^{2}}{\left\|\boldsymbol{s}\left(\tau_{i, j}^{s}\right)\right\|^{2}} .
$$

For a receiver with calibrated power level, the average received signal power is given by $P_{r_{i, j}^{s}}=\frac{1}{N} \sum_{n=1}^{N}\left|r_{i, j}^{s}(n)\right|^{2}$ such that $\operatorname{SNR}\left(r_{i, j}^{s}\right)=\frac{P_{r_{i, j}^{s}}^{s}-\sigma_{r_{i, j}^{s}}^{2}}{\sigma_{r_{i, j}^{s}}^{2 s}}$ is the signal-to-noise ratio (SNR) of the received signal. The noise variance $\sigma_{\boldsymbol{r}_{i}^{s}}^{2}$ can thus be determined from the receiver noise floor. Alternatively, a maximum likelihood (ML) estimator based on the known transmitted signal [43] can be used to estimate the SNR and scale the log-likelihood function (25) appropriately.

2) Multiport Antenna: For nodes with a multiport antenna, $M_{i}>1$, the log-likelihood function of the received signals (3) and (5) is given by:

$$
\begin{aligned}
& L_{i, j}^{s}\left(\phi_{i, j}^{s}, \tau_{i, j}^{s}, \alpha_{i, j}^{s}, \varphi_{i, j}^{s}, \boldsymbol{g}_{i}^{s}\right)=-N \ln \left(\pi \sigma_{\boldsymbol{r}_{i, j}^{s}}^{2}\right) \\
& -\frac{1}{\sigma_{\boldsymbol{r}_{i, j}^{s}}^{2}} \sum_{n=1}^{N}\left\|\boldsymbol{r}_{i, j}^{s}(n)-\boldsymbol{a}\left(\phi_{i, j}^{s}\right) s\left(n, \tau_{i, j}^{s}\right) \alpha_{i, j}^{s} e^{\mathrm{j} \varphi_{i, j}^{s}}\right\|^{2} .
\end{aligned}
$$

Again, plugging the minimum of (26) w.r.t. the unknown signal amplitude and phase back into (26) and neglecting the constant term, see [44], we obtain the concentrated loglikelihood function

$$
\tilde{L}_{i, j}^{s}\left(\phi_{i, j}^{s}, \tau_{i, j}^{s}, \boldsymbol{g}_{i}^{s}\right)=\frac{1}{\sigma_{\boldsymbol{r}_{i, j}^{s}}^{2}}\left\|\frac{\boldsymbol{e}_{i, j}^{s}\left(\boldsymbol{e}_{i, j}^{s}\right)^{H}}{\left(\boldsymbol{e}_{i, j}^{s}\right)^{H} \boldsymbol{e}_{i, j}^{s}} \boldsymbol{r}_{i, j}^{s}\right\|^{2},
$$

with $\boldsymbol{e}_{i, j}^{s}=\operatorname{vec}\left\{\boldsymbol{a}\left(\phi_{i, j}^{s}\right) \boldsymbol{s}\left(\tau_{i, j}^{s}\right)^{T}\right\}$.

\section{SLAC ALGORITHM}

We implement SLAC as a Bayesian filter. The principle of Bayesian filtering is to calculate the posterior probability density function (pdf) $p\left(\boldsymbol{x}^{s} \mid \boldsymbol{z}^{1: s}\right)$ recursively by prediction and update steps [42], [45]. Bayesian filtering for cooperative SLAC poses two challenges. First, the measurement models (25) and (27) are highly nonlinear, which excludes algorithms based on linearization such as the extended Kalman filter (EKF). Second, due to the inclusion of the sampling matrix entries describing the antenna response, the state vector (18) has high dimensionality. For example, for the measurement scenario considered in Section VI, the state vector has 131 entries. This makes the application of particle filtering approaches, which would be well suited for high nonlinearity, challenging. As a compromise, we use an algorithm similar to an iterated extended Kalman filter (IEKF) [45]. By relinearization, the algorithm can cope with high nonlinearities and can also handle a high dimensional state, at the price of computational complexity.

\section{A. Prediction}

For the prediction step, the pdf of the motion model $p\left(\boldsymbol{x}^{s} \mid \boldsymbol{x}^{s^{-}}\right)$is incorporated into the posterior pdf of the previous snapshot, $p\left(\boldsymbol{x}^{s^{-}} \mid \boldsymbol{z}^{1: s^{-}}\right)$, to obtain:

$$
p\left(\boldsymbol{x}^{s} \mid \boldsymbol{z}^{1: s^{-}}\right)=\int p\left(\boldsymbol{x}^{s} \mid \boldsymbol{x}^{s^{-}}\right) p\left(\boldsymbol{x}^{s^{-}} \mid \boldsymbol{z}^{1: s^{-}}\right) \mathrm{d} \boldsymbol{x}^{s^{-}} .
$$

Assuming (28) is approximately Gaussian distributed, we have

$$
p\left(\boldsymbol{x}^{s} \mid \boldsymbol{z}^{1: s^{-}}\right) \approx \mathcal{N}\left(\overline{\boldsymbol{x}}^{s}, \overline{\boldsymbol{\Sigma}}^{s}\right),
$$

with the predicted mean $\overline{\boldsymbol{x}}^{s}$ and covariance $\overline{\boldsymbol{\Sigma}}^{s}$. Prediction for the kinematic states of the agents listed in the agent set $\mathbb{A}$ is done according to the motion model (21),

$$
\overline{\boldsymbol{x}}_{i, \mathrm{loc}}^{s}=\boldsymbol{f}\left(\hat{\boldsymbol{x}}_{i, \mathrm{loc}}^{s^{-}}\right) \quad \forall i \in \mathbb{A},
$$

and the calibration parameter states remain constant, see (14) to (18). Applying linearization as in the EKF, the predicted covariance is

$$
\overline{\boldsymbol{\Sigma}}^{s}=\boldsymbol{F}^{s^{-}} \hat{\boldsymbol{\Sigma}}^{s^{-}}\left(\boldsymbol{F}^{s^{-}}\right)^{T}+\boldsymbol{\Sigma}_{\boldsymbol{x}}^{s}
$$

with the estimated covariance matrix from the previous snapshot $\hat{\boldsymbol{\Sigma}}^{s^{-}}$, the nonzero entries of the Jacobian matrix $\boldsymbol{F}^{s^{-}}$given by

$$
\left[\boldsymbol{F}^{s^{-}}\right]_{\boldsymbol{\iota}_{i, \mathrm{loc}, \boldsymbol{\iota}, \text { loc }}}=\left.\frac{\partial \boldsymbol{f}\left(\boldsymbol{x}_{i, \mathrm{loc}}^{s^{-}}\right)}{\partial \boldsymbol{x}_{i, \mathrm{loc}}^{s^{-}}}\right|_{\boldsymbol{x}_{i, \mathrm{loc}}^{s^{-}}=\hat{\boldsymbol{x}}_{i, \mathrm{loc}}^{s^{-}}},
$$




$$
\left[\boldsymbol{F}^{s^{-}}\right]_{\boldsymbol{\iota}_{i, \mathrm{cal}}, \boldsymbol{\iota}_{i, \mathrm{cal}}}=\left\{\begin{array}{ll}
\mathbb{I}_{2 M_{i} U+1}, & \text { if } M_{i}>1 \\
1, & \text { if } M_{i}=1
\end{array},\right.
$$

and the nonzero entries of the process noise covariance matrix $\boldsymbol{\Sigma}_{\boldsymbol{x}}^{s}$ given by:

$$
\left[\boldsymbol{\Sigma}_{\boldsymbol{x}}^{s}\right]_{\boldsymbol{\iota}_{i, \mathrm{loc}}, \boldsymbol{\iota}_{i, \mathrm{loc}}}=\boldsymbol{\Sigma}_{\boldsymbol{x}_{i, \mathrm{loc}}}^{s}
$$

\section{B. Update}

For the update step of the Bayesian filter, the measurement likelihood $p\left(\boldsymbol{z}^{s} \mid \boldsymbol{x}^{s}\right)$ is taken into account to obtain the posterior pdf of the current snapshot $s$,

$$
p\left(\boldsymbol{x}^{s} \mid \boldsymbol{z}^{1: s}\right)=\frac{p\left(\boldsymbol{z}^{s} \mid \boldsymbol{x}^{s}\right) p\left(\boldsymbol{x}^{s} \mid \boldsymbol{z}^{1: s^{-}}\right)}{p\left(\boldsymbol{z}^{s} \mid \boldsymbol{z}^{1: s^{-}}\right)},
$$

where the denominator is constant. Again assuming that the posterior is approximately Gaussian distributed, the posterior pdf is

$$
p\left(\boldsymbol{x}^{s} \mid \boldsymbol{z}^{1: s}\right) \approx \mathcal{N}\left(\hat{\boldsymbol{x}}^{s}, \hat{\boldsymbol{\Sigma}}^{s}\right)
$$

with mean $\hat{\boldsymbol{x}}^{s}$ and covariance $\hat{\boldsymbol{\Sigma}}^{s}$. Using a maximum a posteriori (MAP) approach, the updated state estimate becomes

$$
\begin{aligned}
& \hat{\boldsymbol{x}}^{s}= \arg \max _{\boldsymbol{x}^{s}} p\left(\boldsymbol{x}^{s} \mid \boldsymbol{z}^{1: s}\right) \\
&=\arg \max _{\boldsymbol{x}^{s}} p\left(\boldsymbol{z}^{s} \mid \boldsymbol{x}^{s}\right) p\left(\boldsymbol{x}^{s} \mid \boldsymbol{z}^{1: s^{-}}\right) \\
&=\arg \max _{\boldsymbol{x}^{s}} \underbrace{\sum_{i \in \mathbb{D}} \ln p\left(\boldsymbol{z}_{i}^{s} \mid \boldsymbol{x}^{s}\right)+\ln p\left(\boldsymbol{x}^{s} \mid \boldsymbol{z}^{1: s^{-}}\right)}_{g\left(\boldsymbol{x}^{s}\right)} \\
&=\arg \max _{\boldsymbol{x}^{s}} \sum_{i \in \mathbb{D}} \sum_{j \in \mathbb{L}_{i}^{s}} L_{i, j}^{s}\left(\boldsymbol{x}_{i}^{s}, \boldsymbol{x}_{j}^{s}\right) \\
& \quad+\frac{1}{2}\left(\boldsymbol{x}^{s}-\overline{\boldsymbol{x}}^{s}\right)^{T}\left(\overline{\boldsymbol{\Sigma}}^{s}\right)^{-1}\left(\boldsymbol{x}^{s}-\overline{\boldsymbol{x}}^{s}\right),
\end{aligned}
$$

where we assume independent process noise for the nodes and independent measurement noise for the signals received by the nodes. For the last equality, the predicted posterior pdf (28) and (29) in logarithm domain is plugged in. By the sum over $\mathbb{L}_{i}^{s}$, only signals which have actually been received for snapshot $s$ are considered, see also the definition of $\mathbb{L}_{i}^{s}$ in Section III-A. The measurement log-likelihood function for a signal sent by node $j$ and received by node $i$,

$$
L_{i, j}^{s}\left(\boldsymbol{x}_{i}^{s}, \boldsymbol{x}_{j}^{s}\right)=\left\{\begin{array}{ll}
\tilde{L}_{i, j}^{s}\left(\phi_{i, j}^{s}, \tau_{i, j}^{s}, \boldsymbol{g}_{i}^{s}\right), & \text { if } M_{i}>1 \\
\tilde{L}_{i, j}^{s}\left(\tau_{i, j}^{s}\right), & \text { if } M_{i}=1
\end{array},\right.
$$

is given by (25) and (27) for singleport and multiport antennas on the receiving node, respectively. A solution to (36) is obtained by the Broyden-Fletcher-Goldfarb-Shanno (BFGS) algorithm, using the gradient

$$
\nabla g\left(\boldsymbol{x}^{s}\right)=\sum_{i \in \mathbb{D}} \sum_{j \in \mathbb{L}_{i}^{s}} \frac{\partial L_{i, j}^{s}\left(\boldsymbol{x}_{i}^{s}, \boldsymbol{x}_{j}^{s}\right)}{\partial \boldsymbol{x}^{s}}+\left(\overline{\boldsymbol{\Sigma}}^{s}\right)^{-1}\left(\boldsymbol{x}^{s}-\overline{\boldsymbol{x}}^{s}\right)
$$

where $\frac{\partial L_{i, j}^{s}\left(\boldsymbol{x}_{i}^{s}, \boldsymbol{x}_{j}^{s}\right)}{\partial \boldsymbol{x}^{s}}$ is derived in Appendix A. BFGS is a quasi-Newton method with proven convergence for convex functions [46]. As problem (36) is in general non-convex, the SLAC algorithm must be initialized with an informative prior $p\left(\boldsymbol{x}^{0}\right)$, which is discussed in the next Section IV-C.
Furthermore, the filter update rate must be chosen according to the system dynamics: With sufficiently high update rate, the position and orientation changes between two consecutive snapshots are small. Thus, the estimated state after prediction $\overline{\boldsymbol{x}}^{s}$, which is used to initialize BFGS, will be close to the estimated state after the update step $\hat{\boldsymbol{x}}^{s}$. The simulation results in Section $\mathrm{V}$ show, that the filter can cope with uncertainty in antenna responses and initializations. In Section VI-C and Fig. 12, we demonstrate with measurement data that the SLAC algorithm converges to approximately the same DoA estimation performance, regardless whether it is initialized with a prior antenna response from a near-field measurement chamber or an EM simulation, although the antenna response from EM simulation deviates considerably from the true antenna response. The result underlines the robustness of the SLAC algorithm regarding a vague antenna response prior.

Using the Laplace approximation [47], the updated covariance matrix is given by the inverse of the Hessian matrix at the solution,

$$
\left[\hat{\boldsymbol{\Sigma}}^{s}\right]_{m, n}=\left(\left.\frac{\partial^{2} g\left(\boldsymbol{x}^{s}\right)}{\partial\left[\boldsymbol{x}^{s}\right]_{m} \partial\left[\boldsymbol{x}^{s}\right]_{n}}\right|_{\boldsymbol{x}^{s}=\hat{\boldsymbol{x}}^{s}}\right)^{-1}
$$

which is evaluated numerically.

\section{Prior}

The Bayesian filter is initialized with a snapshot estimate of the agent positions $\hat{\boldsymbol{p}}_{i}^{0}=\left[\hat{x}_{i}^{0}, \hat{y}_{i}^{0}\right]^{T}$ and orientations $\hat{\psi}_{i}^{0}$ with variances $\sigma_{\boldsymbol{p}^{0}}^{2}$ and $\sigma_{\psi^{0}}^{2}$. One of the algorithms [48]-[50] can be used to obtain such a snapshot estimate based on the estimated distances and DoAs. We assume that initially, the agents are not moving, such that $\hat{v}_{i}^{0}=0 \mathrm{~m} / \mathrm{s}$ and $\hat{\omega}_{i}^{0}=0 \%$. As the ranging bias is expected to be small compared to the actual distances, we initialize it with $\hat{\delta}_{i}^{0}=0 \mathrm{~m}$. As mentioned in Section II, prior knowledge regarding the antenna response(s) is available in terms of the sampling matrix entries $\hat{\boldsymbol{g}}_{i}^{0}$. This prior knowledge could come for instance from EM simulations or antenna measurements in a near-field measurement chamber. Thus for every node $i \in \mathbb{D}$ we have a Gaussian prior

$$
p\left(\boldsymbol{x}_{i}^{0}\right)=(2 \pi)^{-\frac{5}{2}} \operatorname{det}\left(\hat{\boldsymbol{\Sigma}}_{i}^{0}\right)^{-\frac{1}{2}} e^{-\frac{1}{2}\left(\boldsymbol{x}_{i}^{0}-\hat{\boldsymbol{x}}_{i}^{0}\right)^{T} \hat{\boldsymbol{\Sigma}}_{i}^{0}\left(\boldsymbol{x}_{i}^{0}-\hat{\boldsymbol{x}}_{i}^{0}\right)}
$$

with mean

$$
\hat{\boldsymbol{x}}_{i}^{0}=\left[\begin{array}{llllll}
\left(\hat{\boldsymbol{p}}_{i}^{0}\right)^{T} & \hat{v}_{i}^{0} & \hat{\psi}_{i}^{0} & \hat{\omega}_{i}^{0} & \hat{\delta}_{i}^{0} & \left(\hat{\boldsymbol{g}}_{i}^{0}\right)^{T}
\end{array}\right]^{T}
$$

and covariance matrix

$$
\hat{\boldsymbol{\Sigma}}_{i}^{0}=\operatorname{diag}\left\{\left[\begin{array}{llllll}
\mathbf{1}_{2} \sigma_{\boldsymbol{p}^{0}}^{2} / 2 & \sigma_{v^{0}}^{2} & \sigma_{\psi^{0}}^{2} & \sigma_{\omega^{0}}^{2} & \sigma_{\delta^{0}}^{2} & \mathbf{1}_{2 M U} \sigma_{\boldsymbol{g}^{0}}^{2}
\end{array}\right]\right\} .
$$

\section{Complexity}

For a worst-case asymptotic complexity analysis we assume a fully connected network, where all nodes are agents, thus $\mathbb{D}=\mathbb{A}$. Each agent is equipped with a multiport antenna with $M$ ports, described by $U$ basis functions, see (10) and (11), which results in a state vector of dimension $\boldsymbol{x}^{s} \in$ $\mathbb{R}^{|\mathbb{D}|(6+2 M U)}$. Complexity is dominated by the update step of the Bayesian filter, where one iteration of BFGS, including 


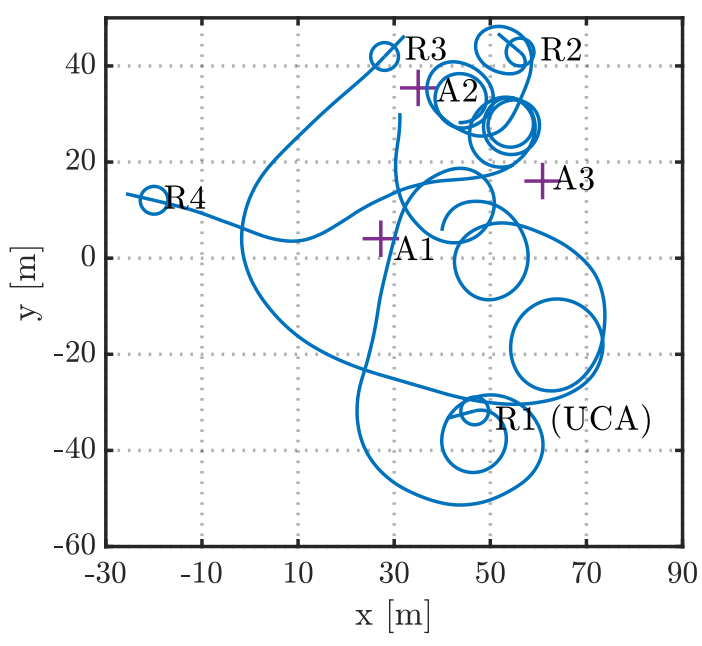

Fig. 2. Example simulation scenario with anchors A1, A2, A3 and randomly generated trajectories for robotic agents R1 (UCA), R2, R3, R4.

the calculation of the log-likelihoods (27) and gradient (38) is $\mathcal{O}\left((|\mathbb{D}| M U)^{2}+|\mathbb{D}|^{2} M N\right)$. Calculation of the Hessian in (39) is $\mathcal{O}\left((|\mathbb{D}| M U)^{2}\right)$ and the matrix inverse in (36) and (39) is $\mathcal{O}\left((|\mathbb{D}| M U)^{3}\right)$ in practice.

\section{Simulation Results}

\section{A. Simulation Setup}

First we evaluate the performance of the proposed SLAC algorithm by simulation. For a fair comparison, we apply the same algorithm derived in Section IV to both SLAC and localization-only, where for localization-only, the calibration states (16) are not part of the state vector. We assume a network with seven nodes: three anchors A1, A2, A3 and four moving agents R1, R2, R3, R4. The agent R1 is equipped with a uniform circular array (UCA) with $M_{i}=4$ antenna elements. The other agents and the anchors are assumed to have singleport antennas. In order to demonstrate that SLAC does not only work for a specific scenario, we have performed a Monte Carlo simulation with 100 runs. For each run, random trajectories are generated for all agents according to the motion model (20), (21) and (23). For the process noise of the motion model (23), we assume $\sigma_{\dot{v}}=0.015 \mathrm{~m} / \mathrm{s}^{1.5}$ for the linear and $\sigma_{\dot{\omega}}=0.3^{\circ} / \mathrm{s}^{1.5}$ for the angular velocity, respectively, and an update interval of $T=0.1 \mathrm{~s}$. One realization of the trajectories is shown in Fig. 2. The UCA sampling matrix with $U=9$ basis functions is obtained by (13), where $\boldsymbol{E}_{i}^{0}$ consists of the UCA steering vector, see e.g. [37], evaluated at a fixed grid $\phi=\left[\phi_{1}, \ldots, \phi_{Q}\right]$. To show that SLAC can cope with different antenna responses, a random antenna response is generated for each Monte Carlo run by distorting the UCA sampling matrix, $\boldsymbol{G}_{i}=\boldsymbol{G}_{\mathrm{UCA}}+\boldsymbol{W}_{\boldsymbol{G}}$ with $\boldsymbol{W}_{\boldsymbol{G}}=\left[\boldsymbol{w}_{\boldsymbol{g}}^{1}, \ldots, \boldsymbol{w}_{\boldsymbol{g}}^{U}\right]$, $\boldsymbol{w}_{\boldsymbol{g}}^{u} \sim \mathcal{C} \mathcal{N}\left(0, \sigma_{\boldsymbol{g}}^{2} \mathbb{I}_{M_{i}}\right)$ and $\sigma_{\boldsymbol{g}}=0.2$. The ranging biases are generated as $\delta_{i}=\mathcal{N}\left(0, \sigma_{\delta}^{2}\right)$ with $\sigma_{\delta}=0.2 \mathrm{~m}$. The transmitted signals and the simulation parameters are according to the measurement setup from Section VI-A: We assume $1.68 \mathrm{GHz}$ carrier frequency, $-15 \mathrm{dBm}$ transmit power, $2 \mathrm{dBi}$ antenna gain, $290 \mathrm{~K}$ receiver noise temperature, $8 \mathrm{~dB}$ noise figure and free-space path loss. The standard deviations of the

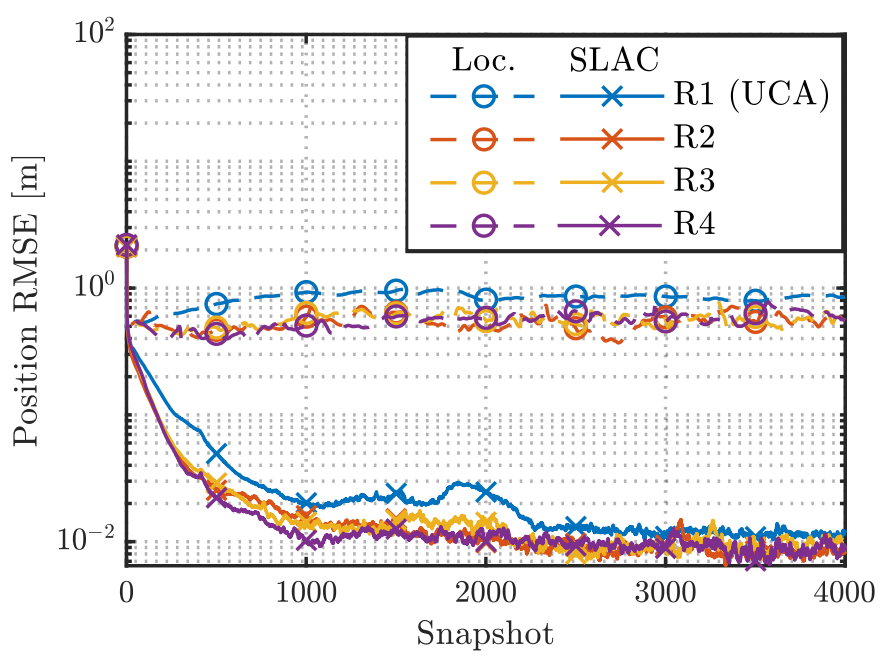

(a) Position RMSE.

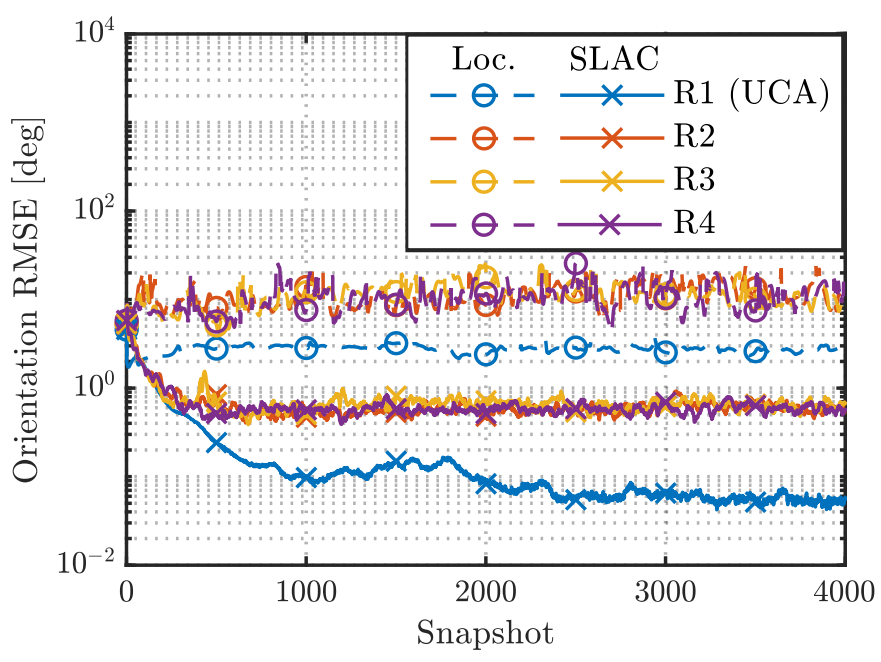

(b) Orientation RMSE.

Fig. 3. Simulated position and orientation RMSE over 100 random trajectories for agents R1, R2, R3, R4. Dashed lines refer to localization-only, solid lines to SLAC.

Gaussian prior (40) are chosen as $\sigma_{p^{0}}=2 \mathrm{~m}, \sigma_{v}^{0}=0.01 \mathrm{~m} / \mathrm{s}$, $\sigma_{\psi^{0}}=7.5^{\circ}, \sigma_{\omega^{0}}=0.01 \%, \sigma_{\delta^{0}}=0.01 \sigma_{\delta}$ and $\sigma_{g^{0}}=0.01 \sigma_{\boldsymbol{g}}$. A low prior covariance is chosen for the ranging biases and the sampling matrix entries, to ensure slow convergence and avoid too fast convergence into local minima. For the simulation, the position and orientation states of the agents as well as the sampling matrix entries are initialized randomly by sampling from the Gaussian prior (40). Ranging bias and velocity states are initialized as described in Section IV-C.

\section{B. Position and Orientation Estimation}

We start by comparing the position root-mean-square error (RMSE) of localization-only and SLAC depicted in Fig. 3a. Initially, the position RMSE is dictated by the prior. For localization-only, the position RMSE quickly decreases with the first snapshots and then stays relatively constant in the order of $0.4 \mathrm{~m}$ to $1.0 \mathrm{~m}$. With SLAC, the RMSE decreases during the first 1000 snapshots to $\mathrm{cm}$ level, as the calibration parameters are estimated. A small improvement is visible up 
to snapshot 2000, as calibration parameters are refined. After that, the RMSE stays relatively constant. For localization-only and in the first part also for SLAC, the position RMSE of the multiport agent R1 with UCA is slightly worse compared to agents R2, R3 and R4 with singleport antennas. Apparently, in the case of a model mismatch of the antenna response, DoA information could do more harm than good. In summary, for the assumed ranging biases and antenna response deviations motivated by practical experience, the position RMSE with SLAC is more than one order of magnitude lower compared to localization-only.

Next, we take a look at the simulated orientation RMSE in Fig. 3b. For localization-only, the orientation RMSE of the agents R2, R3 and R4 with singleport antennas is in the order of $5^{\circ}$ to $25^{\circ}$ and appears noisy. In fact, agents R2, R3 and R4 cannot observe their orientation directly. The orientation information is only obtained over time via the motion model (21) by movement of the agents and subsequent position changes. Being only indirectly observable, the estimated orientations are noisy. The orientation RMSE of agent R1 with is lower, around $3^{\circ}$, as the orientation can be observed not only through the motion but also by the four element UCA. With SLAC, the orientation RMSE of R2, R3 and R4 decreases to sub-degree level within the first 500 snapshots. As the ranging biases are calibrated, ranging information becomes more accurate, leading to better position accuracy and thus also better orientation accuracy, which is estimated through position differences. The orientation RMSE of R1 decreases even further, as the UCA is calibrated. After 2000 snapshots, the orientation RMSE of R1 (UCA) has reached values below $0.1^{\circ}$ and stays constant as well. For the assumed ranging biases and antenna response deviations, the orientation RMSE improves by more than one order of magnitude with SLAC. Although both show considerable improvements, the relative improvement in the position domain is larger compared to the orientation domain.

Usually, position and orientation errors are the figures of interest for a localization system. However, they are influenced by many aspects such as geometry, SNR, motion model and the choice of parameters. For that reason, we have a closer look on the antenna and ranging bias calibration.

\section{Antenna Calibration}

We evaluate the quality of antenna calibration by the DoA estimation performance. For that, we simulate ML DoA estimation for a discrete set of DoAs $\phi_{q}$ with $q \in\{1, \ldots, Q\}$ and $Q=360$ spanning a regular grid with step size $1^{\circ}$ over the whole manifold. Fig. 4 shows the DoA estimation RMSE over this grid and over the 100 different trajectories and antenna responses, see Section V-A. The ML estimator uses the prior antenna response, $\hat{\boldsymbol{a}}_{i}^{0}(\phi)=\hat{\boldsymbol{G}}_{i}^{0} \boldsymbol{b}(\phi)$, and the antenna response estimated by SLAC, $\hat{\boldsymbol{a}}_{i}^{s}(\phi)=\hat{\boldsymbol{G}}_{i}^{s} \boldsymbol{b}(\phi)$, respectively. Using the prior antenna response, we obtain an RMSE of approx. $11^{\circ}$ due to the model mismatch. With SLAC, the DoA RMSE initially increases slightly, while the estimated antenna response is adjusted. Then it decreases and from snapshot 2200 on, sub-degree accuracy is reached. The

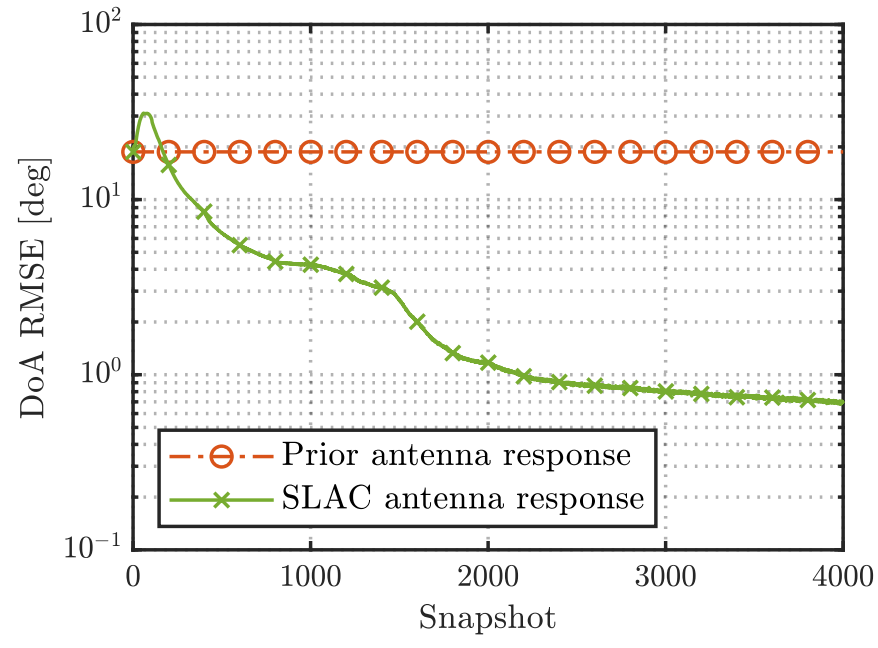

Fig. 4. ML DoA estimation RMSE over entire manifold and over 100 random trajectories and random antenna responses. The ML estimator uses the prior antenna response $\hat{\boldsymbol{a}}_{i}^{0}(\phi)=\hat{\boldsymbol{G}}_{i}^{0} \boldsymbol{b}(\phi)$ and the antenna response estimated by SLAC $\hat{\boldsymbol{a}}_{i}^{s}(\phi)=\hat{\boldsymbol{G}}_{i}^{s} \boldsymbol{b}(\phi)$, respectively.

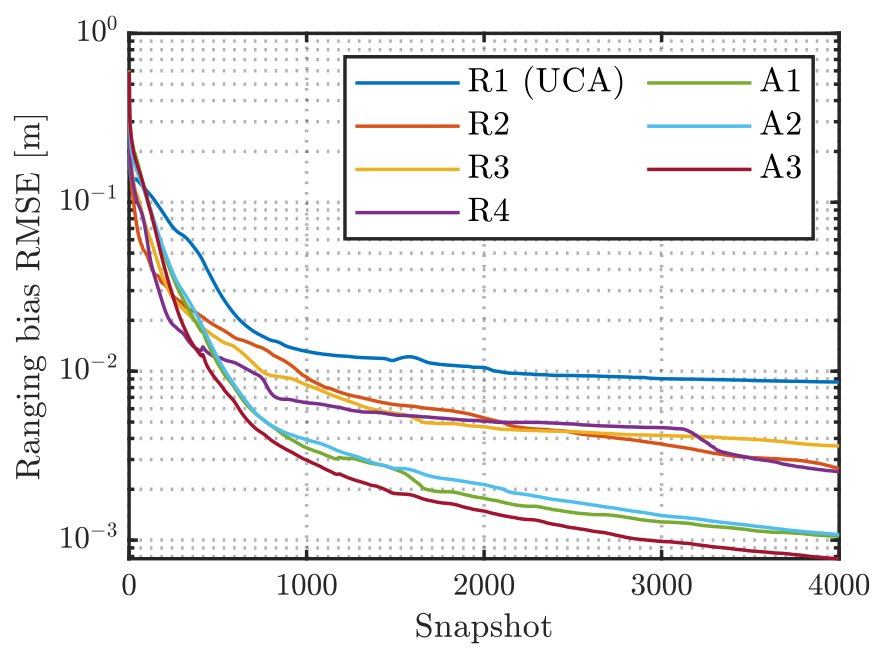

Fig. 5. Simulated ranging bias RMSEs over 100 random trajectories for agents R1, R2, R3, R4 and anchors A1, A2, A3.

continuous improvement shows that the estimated antenna response converges and model mismatch is reduced with increasing number of snapshots. We conclude that the antenna response can be calibrated by SLAC, thus improving the DoA estimation performance.

\section{Ranging Bias Calibration}

Next we investigate the performance of the ranging bias calibration by SLAC. Fig. 5 shows the ranging bias RMSE over the 100 random trajectories. The ranging biases are in the order of decimeters, see Section V-A. As the prior for the ranging bias estimation is zero, see (40), the initial ranging bias RMSEs are in the order of the ranging biases. During the first snapshots, the ranging bias RMSEs of all nodes decrease quickly. Then the curves flatten and the ranging bias RMSEs decrease more slowly, reaching sub-centimeter levels after 2100 snapshots. Interestingly, the curves are separated into 


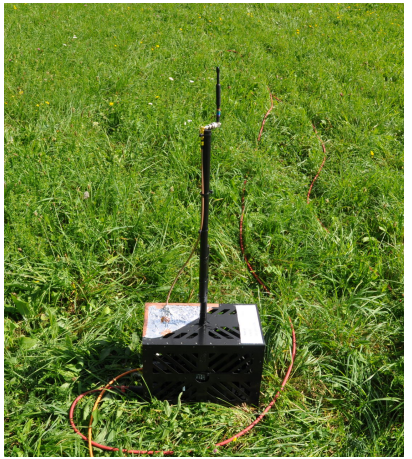

(a) Anchor node.

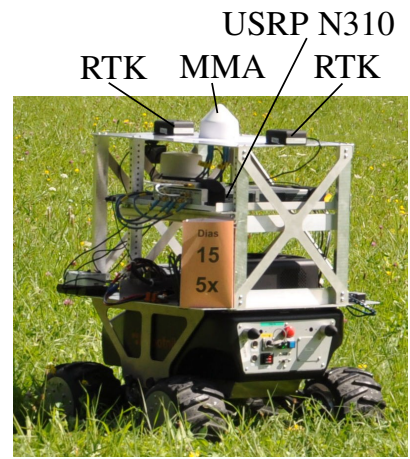

(b) Agent Dias with MMA.
Fig. 6. Pictures of an anchor node and agent Dias, a robotic rover

three distinct groups. The ranging bias RMSEs of the anchors A1, A2, A3 reach the lowest values, which is explained by their known position. For the agents with singleport antennas $\mathrm{R} 2, \mathrm{R} 3, \mathrm{R} 4$, only the ranging bias needs to be estimated, compared to the multiport antenna agent R1 (UCA), where also the antenna response is estimated simultaneously. This leads to lower ranging bias RMSE for R2, R3, R4, compared to $\mathrm{R} 1$. In conclusion, the ranging biases can be calibrated by SLAC for the simulated trajectories, which improves distance information and contributes to enhanced position and orientation performance.

\section{Measurement Results}

\section{A. Measurement Setup}

As SLAC is motivated by practical considerations, an important contribution of this paper is a performance evaluation with measurement data. The measurement setup consists of three anchor nodes, see Fig. 6a, and four robotic rovers named Dias, Drake, Magellan and Vespucci. The anchor nodes as well as Drake, Magellan and Vespucci are equipped with a dipole antenna. On Dias, an MMA with four ports is installed, see Fig. 6b. The MMA, which has been developed in-house at the German Aerospace Center (DLR), is a dielectric resonator antenna with four independently excited modes [51]. DoA estimation with this MMA has been shown in [52]. For the manifold separation (10), $U=13$ basis functions (11) are chosen to represent the antenna response. A commercial dual antenna real-time kinematic (RTK) system [53], which internally also uses inertial sensors, is installed on all rovers to provide ground-truth.

The physical layer signaling is based on our in-house developed DLR Swarm Communication and Navigation system [52], [54]. The system parameters are $1.68 \mathrm{GHz}$ carrier frequency, $31.25 \mathrm{MHz}$ sampling rate, $-15 \mathrm{dBm}$ transmit power and orthogonal frequency-division multiplexing (OFDM) with fast Fourier transform (FFT) length 1024. The transmitted baseband signals are Zadoff-Chu sequences of length $N=463$ mapped onto 925 subcarriers, where every second subcarrier is occupied, resulting in an occupied bandwidth of approx. $28.2 \mathrm{MHz}$. A unique sequence is assigned to each node, where sequences with low cross-correlation are chosen. The system is implemented as SDR, where Ettus Research Universal

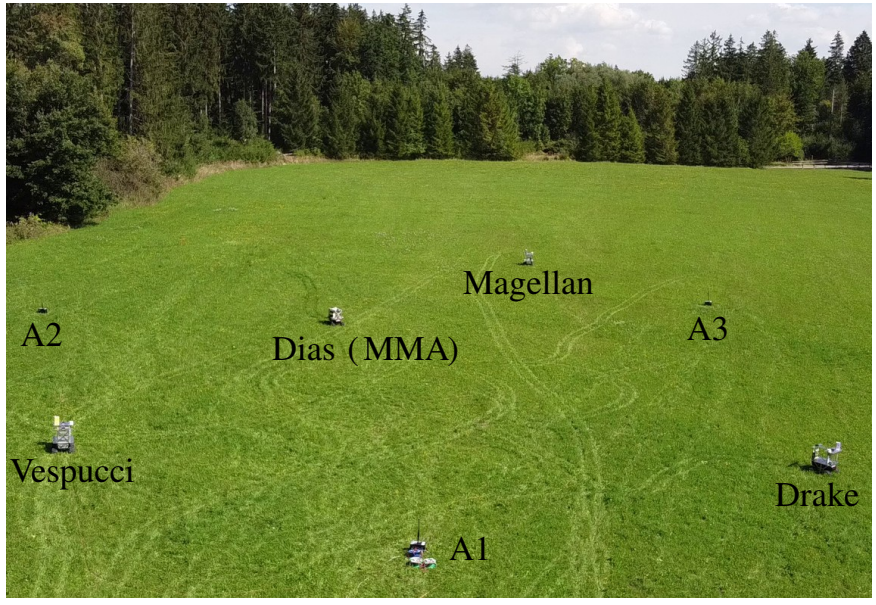

Fig. 7. Bird's-eye view of the measurement scenario showing anchors A1, A2, A3 and agents Dias (MMA), Drake, Magellan, Vespucci.

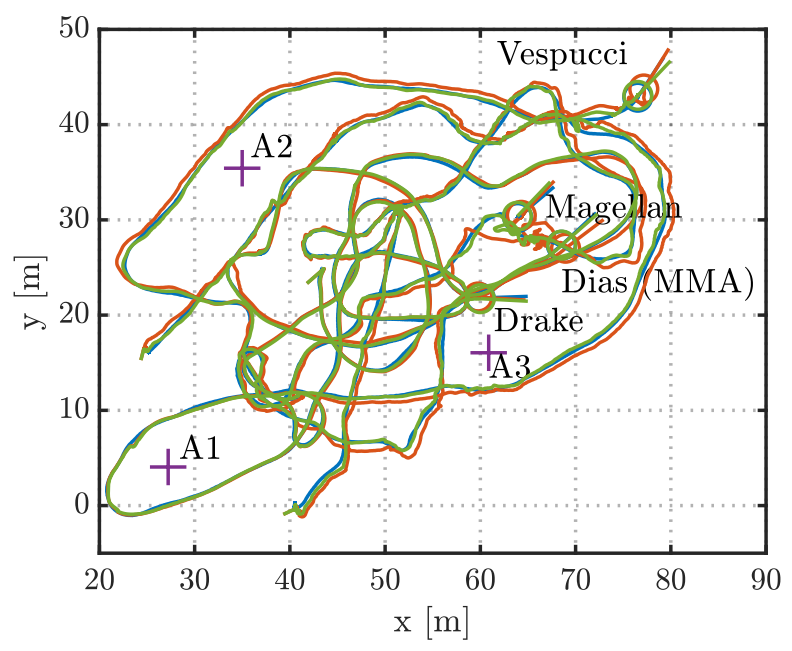

Fig. 8. Anchor positions and trajectories of robotic rover agents. Ground truth is plotted in blue, localization-only in orange and SLAC in green.

Software Radio Peripheral (USRP) B200mini devices are used for the nodes with singleport antennas. On Dias, the USRP N310 from Ettus Research with four transceiver channels is installed. The local oscillator (LO) provided by an external frequency synthesizer, which allows phase-coherent reception on four channels. Phase and amplitude imbalances between the channels have been calibrated before the experiment. The receiver is thus prepared for DoA estimation with the MMAs, where both magnitude and phase of the received signal provide DoA information, see [13], [52] and Fig. 10.

The experiment was conducted on open grassland. The site with the positions of the three anchor nodes and the initial positions of the robotic rovers is shown in Fig. 7. The total duration of the experiment was $12 \mathrm{~min} 30 \mathrm{~s}$. First only Dias (MMA) was driving. At about 6 min $0 \mathrm{~s}$, Drake and Vespucci started driving as well. Magellan remained static for the whole time.

During the experiment, the received signals have been stored and evaluated in post-processing with the proposed SLAC algorithm and localization-only for comparison. The algorithm 


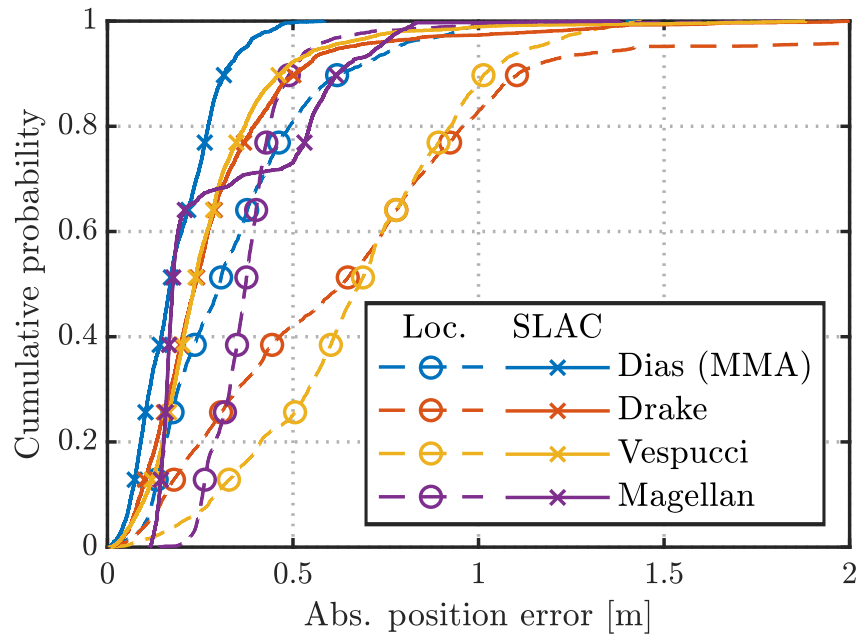

(a) $\mathrm{CDF}$ of the absolute position error.

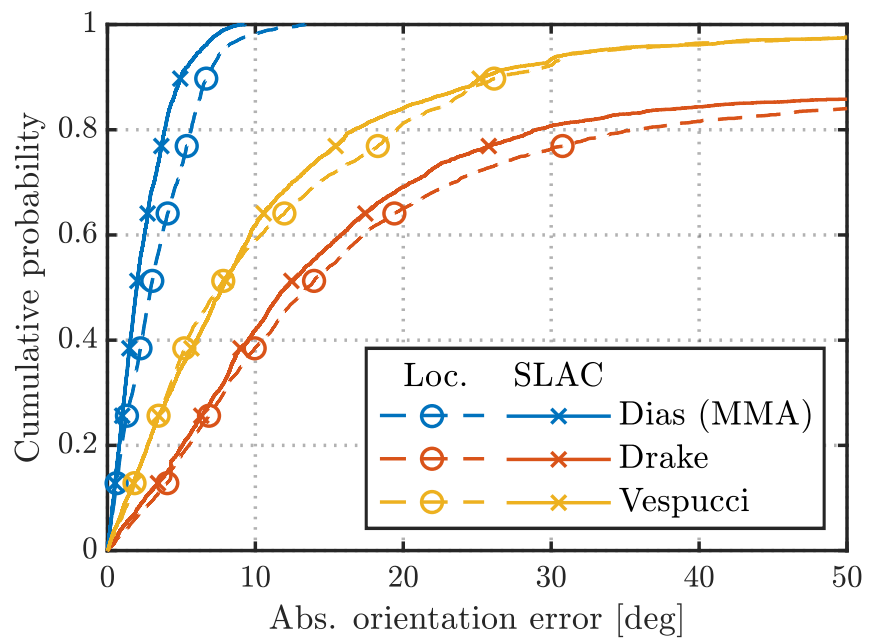

(b) $\mathrm{CDF}$ of the absolute orientation error.

Fig. 9. Empirical CDFs of the absolute position error $\left\|\hat{\boldsymbol{p}}_{i}^{s}-\boldsymbol{p}_{i}^{s}\right\|$ and absolute orientation error $\left|\hat{\psi}_{i}^{s}-\psi_{i}^{s}\right|$ of the agents for localization-only and SLAC.

parameters are identical to the simulation, see Section V-A. To account for real-world effects, for instance shaking rovers moving on rough terrain, the noise standard deviation $\sigma_{\boldsymbol{r}_{i, j}^{s}}$ is multiplied by two, see (3). SNR of the received signals varies between $3 \mathrm{~dB}$ to $27 \mathrm{~dB}$. The ground-truth trajectories as well as the estimated trajectories for localization-only and SLAC are shown in Fig. 8. Hereafter, we examine position and orientation estimation performance, as well as the antenna and ranging bias calibration. To provide a fair comparison, the Bayesian filtering algorithm derived in Section IV is applied to both SLAC and localization-only, where for localization-only, the calibration states (16) are omitted from the state vector.

\section{B. Position and Orientation Estimation}

Position and orientation estimation performance is evaluated for the second part of the measurements, from $6 \min 0 \mathrm{~s}$ to $12 \mathrm{~min} 30 \mathrm{~s}$, when all agents except Magellan are moving. First we focus on positioning. An empirical CDF of the absolute position error of all agents is shown in Fig. 9a. For localizationonly, the $90^{\text {th }}$ percentile error is larger than $1 \mathrm{~m}$ for Drake and Vespucci, and $0.62 \mathrm{~m}$ and $0.49 \mathrm{~m}$ for Dias (MMA) and Magellan, respectively. With SLAC, positioning performance is improved considerably, lowering the $90^{\text {th }}$ percentile error to $0.51 \mathrm{~m}, 0.47 \mathrm{~m}$ and $0.32 \mathrm{~m}$ for Drake, Vespucci and Dias. As Magellan is not moving during the whole time, parameter observability is impaired and its ranging bias is estimated incorrectly at the beginning, see Fig. 13. This causes some amount of snapshots with larger position errors and the outlier in the CDF curve Fig. 9a. The positioning performance for Dias with MMA is slightly better than for Drake and Vespucci with singleport antennas. Overall, cooperative SLAC shows a considerable improvement in positioning performance, which confirms the simulation results.

Next, we investigate the empirical CDF of the absolute orientation error shown in Fig. 9b. For Dias with the fourport MMA, the $90^{\text {th }}$ percentile error decreases from $6.7^{\circ}$ with localization-only to $5.0^{\circ}$ with SLAC. Drake and Vespucci are equipped with a singleport antenna, their orientation can thus only be observed indirectly through position changes over time and the motion model (21). This results in much larger orientation errors, e.g. $16 \%$ of Drake's orientation errors are $>50^{\circ}$. The comparably bad orientation estimation performance of singleport agents is also apparent in the simulation, see Fig. 3b, but the effect is more pronounced for the measurement data. However, in a practical system, one would usually include other sensors to alleviate this problem, see the discussion in Section VII-C. In general, SLAC improves the orientation estimation performance, especially for the multiport agent Dias with MMA. However, the improvement is not as pronounced as in the position domain. Potential causes could be more accurate prior information, or a mismatch of the motion model.

Also for the measurement results, the position and orientation performance is influenced by many different aspects like geometry, propagation and SNR, the motion model and the choice of parameters. In order to investigate the calibration aspect more deeply, we treat the antenna and ranging bias calibration using the measurement data separately.

\section{Antenna Calibration}

Fig. 10 shows the antenna response of the four-port MMA installed on Dias in terms of its power and phase pattern. Before it was integrated on the rover, the MMA antenna response has been measured in a near-field measurement chamber. The measured antenna response $\hat{\boldsymbol{a}}_{i}^{0}(\phi)=\hat{\boldsymbol{G}}_{i}^{0} \boldsymbol{b}(\phi)$, see (12a), (12b) and (13), has then been used for the localization algorithm and as prior for SLAC. The shape of the final antenna response estimated by SLAC $\hat{\boldsymbol{a}}_{i}^{S}(\phi)=\hat{\boldsymbol{G}}_{i}^{S} \boldsymbol{b}(\phi)$ is similar to the measurement from the near-field measurement chamber. However, there are differences visible in both, power and phase domain.

To evaluate the impact of the antenna response calibration, all signals received by the MMA on Dias from its neighboring nodes are used to perform ML DoA estimation [13], [37] in post-processing. Fig. 11 shows the empirical CDF curves of the absolute ML DoA estimation error $\left|\hat{\phi}_{i, j}^{s}-\phi_{i, j}^{s}\right|$, using either the prior antenna response or the estimated antenna response by SLAC. For all neighbors, DoA estimation performance with 

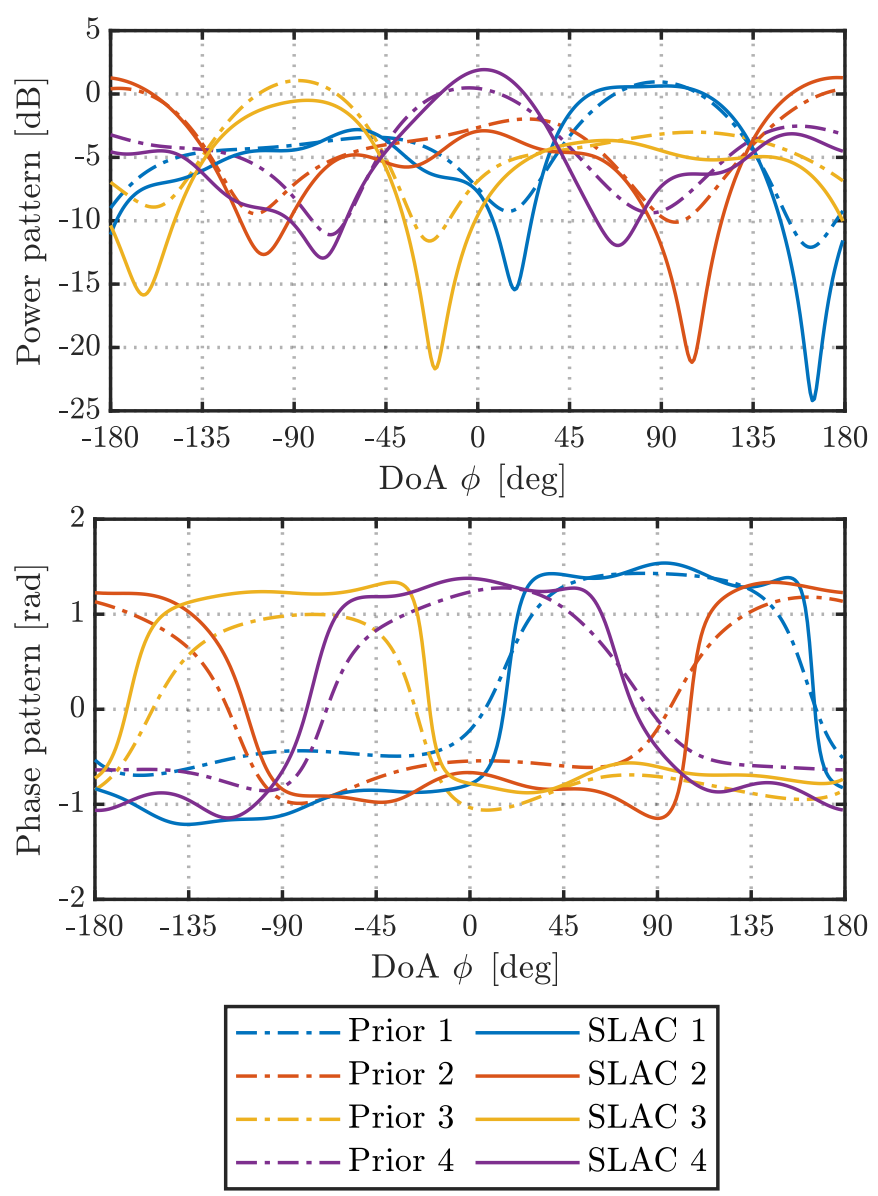

Fig. 10. Prior antenna response $\hat{\boldsymbol{a}}_{i}^{0}(\phi)=\hat{\boldsymbol{G}}_{i}^{0} \boldsymbol{b}(\phi)$ and antenna response estimated by SLAC after $S$ snapshots $\hat{\boldsymbol{a}}_{i}^{S}(\phi)=\hat{\boldsymbol{G}}_{i}^{S} \boldsymbol{b}(\phi)$ of the MMA with four ports installed on rover Dias. The prior antenna response obtained in a near-field measurement chamber is represented by dashed-dotted and the antenna response estimated with SLAC by solid lines.

the SLAC antenna response outperforms the prior antenna response, as the curves move towards the top left of the plot. With the prior antenna response, the $90^{\text {th }}$ percentile DoA estimation error is between $7.0^{\circ}$ to $11.3^{\circ}$, whereas with SLAC it is between $4.1^{\circ}$ to $6.3^{\circ}$. Over all agents, this means an improvement from $9.6^{\circ}$ to $5.9^{\circ}$. In contrast to the near-field measurement, where only the antenna itself can be measured, SLAC is able to estimate the installed antenna response on the rover. The model mismatch of the antenna response is reduced and DoA estimation performance is improved.

In order to analyze the cooperation gain for antenna calibration, we compare non-cooperative, where no agent to agent links are present, to cooperative SLAC. Fig. 12 shows again the empirical CDF of the absolute DoA estimation error, but now for signals received by the MMA of Dias. Using the prior antenna response obtained in the near-field measurement chamber, cooperative SLAC is slightly better than non-cooperative SLAC. We further examine a second case, where the antenna response prior is less accurate, by using the antenna response from EM simulation of the MMA as prior and assuming $\sigma_{\boldsymbol{g}}=0.3$. As EM simulation does not account for manufacturing inaccuracies, DoA estimation performance with the antenna response from EM simulation

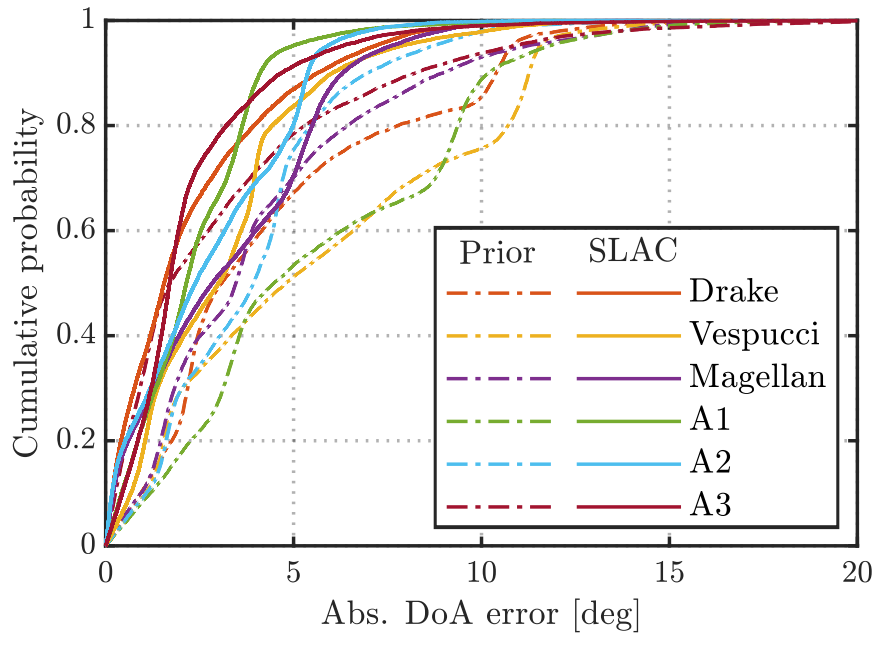

Fig. 11. Empirical CDF of the absolute DoA estimation error $\left|\hat{\phi}_{i, j}^{s}-\phi_{i, j}^{s}\right|$ for the signals received by Dias (MMA) per transmitting node. Dashed lines represent DoA estimation using the prior antenna response from a near-field measurement chamber, solid lines with the antenna response estimated by SLAC.

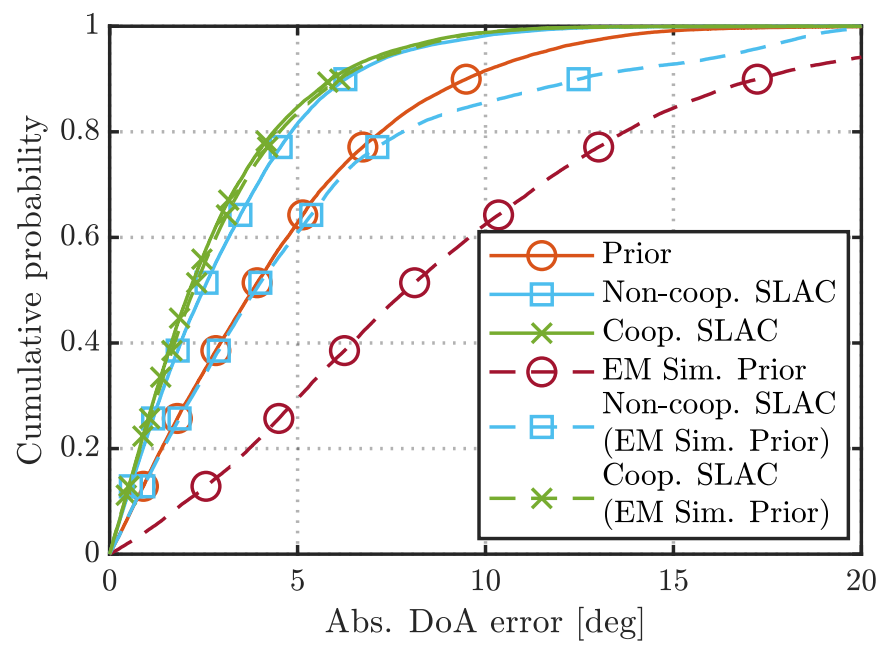

Fig. 12. Empirical CDF of the absolute DoA estimation error for signals received by Dias (MMA), using the prior antenna response from a near-field measurement chamber (Prior) and the antenna response from non-cooperative and cooperative SLAC. A second case with the prior antenna response from EM simulation (EM Sim. Prior) is shown with dashed lines.

is clearly worse. In this case, non-cooperative SLAC can improve the performance, but cannot surpass the prior from the near-field measurement chamber. Cooperative SLAC is able to achieve almost the same performance as with the more accurate prior from the near-field measurement chamber. By cooperation, more signals from more diverse directions are received and position and orientation accuracy is improved [14]-[18], which leads to faster convergence of the antenna response calibration.

\section{Ranging Bias Calibration}

Fig. 13 shows the estimated ranging biases $\delta_{i}^{s}$ of all agents and anchors over time. From the prior value of $0 \mathrm{~m}$, they quickly converge to initial estimates. After around $3 \mathrm{~min}$, the 


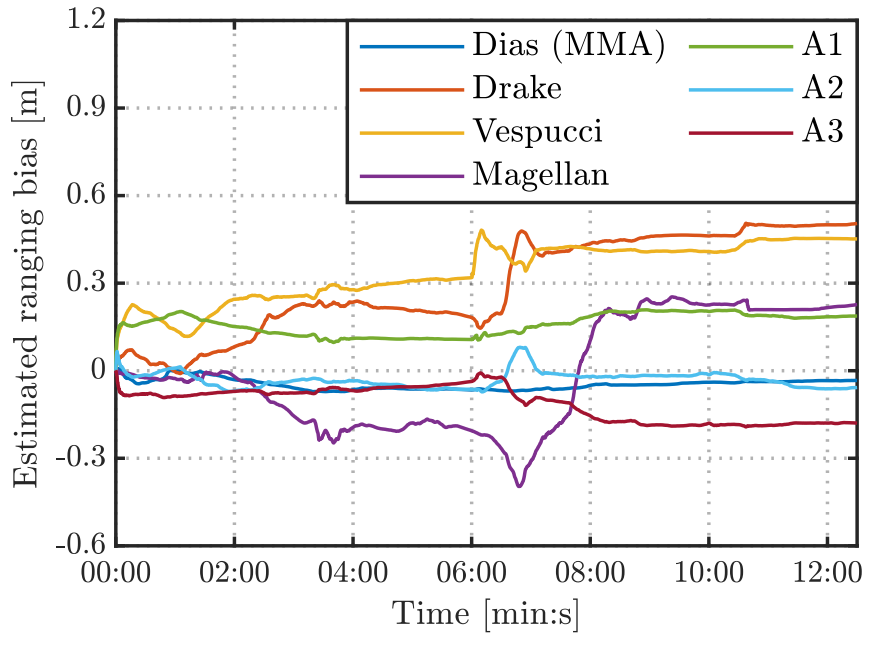

Fig. 13. Estimated ranging biases $\hat{\delta}_{i}^{s}$ for all agents and anchors over time.

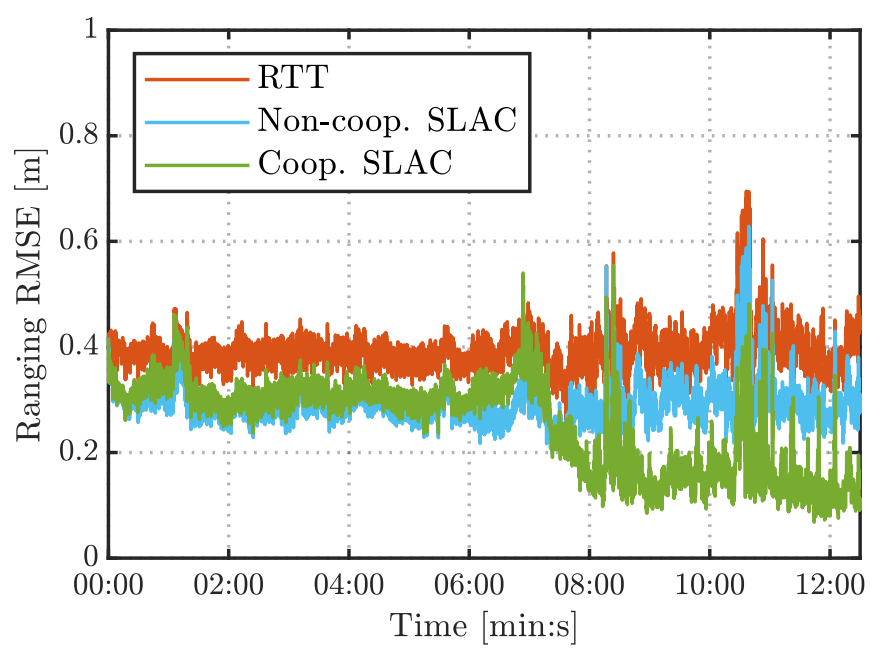

Fig. 14. Ranging RMSE over all links in the network without correction and with non-cooperative and cooperative SLAC over time.

estimates are refined due to the movement of Dias (MMA) and stay constant until 6 min. Then, when Drake and Vespucci start moving, especially the ranging biases of Drake and Magellan change again. From $8 \mathrm{~min}$ on, the estimations have converged and stay relatively constant until the end. The plot underlines the importance of motion for the parameter observability. When only one agent is moving, the ranging biases are only partially observable.

In order to evaluate if estimating the ranging biases has improved ranging accuracy, we calculate the ranging RMSE over all links in the network, $\sqrt{\sum_{i \in \mathbb{D}}\left(\sum_{j \in \mathbb{L}_{i}^{s}}\left|\hat{d}_{i, j}^{s}-d_{i, j}^{s}\right|^{2} /\left|\mathbb{L}_{i}^{s}\right|\right) /|\mathbb{D}|}$. Using the signal round-trip time (RTT), the estimated distance without correction is calculated as $\hat{d}_{i, j}^{s}=c\left(\hat{\tau}_{i, j}^{s}-\bar{\tau}_{i, j}^{s}\right) / 2$, and with ranging bias correction by SLAC as $\hat{d}_{i, j}^{s}=c\left(\hat{\tau}_{i, j}^{s}-\bar{\tau}_{i, j}^{s}\right) / 2+\hat{\delta}_{i}^{s}+\hat{\delta}_{j}^{s}$, see (1). The ranging RMSEs without ranging bias correction and with correction by non-cooperative and cooperative SLAC are shown in Fig. 14. Without correction, the ranging RMSE is usually around $0.4 \mathrm{~m}$, except towards the end of the experiment, where larger peaks occur. Until $5 \mathrm{~min} 50 \mathrm{~s}$, non-cooperative and cooperative SLAC show a similar behavior: The ranging RMSE quickly decreases to around $0.3 \mathrm{~m}$, then it stays relatively constant. Next, there is a short period, where cooperative SLAC is slightly worse. Looking at Fig. 13, this can be explained by the estimated ranging bias of the static agent Magellan, which is first estimated with the wrong sign. When three agents are moving, the algorithm is able to correct the mistake and from 8 min until $12 \mathrm{~min}$ $30 \mathrm{~s}$, the ranging RMSE with cooperative SLAC is mostly below $0.2 \mathrm{~m}$. The peaks towards the end are likely caused by larger distances between the agents and anchors. Without cooperation, the ranging biases are not fully observable, and the ranging RMSE remains around $0.3 \mathrm{~m}$. The considerable decrease of the ranging RMSE from $0.4 \mathrm{~m}$ to $0.2 \mathrm{~m}$ using cooperative SLAC emphasizes that cooperative SLAC is able to estimate ranging biases and thus improve ranging accuracy.

\section{DISCUSSION}

\section{A. Observability}

A detailed observability analysis of SLAC for cooperative localization is an open research topic, which is beyond the scope of this paper. However, we discuss a few initial observations. For the antenna calibration aspect, it is intuitive that the full manifold should be covered, as the antenna response cannot be estimated for directions, from which no signal has arrived. However, since the antenna response is spatially bandlimited [38], [39], it is not necessary to observe signals on an infinitesimally small angular grid. Applied to cooperative SLAC, this means the antenna response is observable when the respective agent is doing turns, or another agents drives around it. In practice, the update interval is usually chosen such that it is suitable for the envisaged agent dynamics. Signals should thus be observed on a small enough angular grid when the robot is turning. If this is not the case, then simply more turns are necessary to capture the antenna response. For the ranging bias calibration, we also notice the importance of motion. For instance in Fig. 13, we see that when only one agent in the network is moving, the ranging bias of Magellan is estimated wrongly. With three moving agents, all ranging biases become observable and are estimated correctly.

\section{B. Multipath Propagation}

The proposed SLAC algorithm directly evaluates the likelihood functions of the received signals (25) and (27) as in direct localization [55]. This is in contrast to the two step approach, where first directions and distances are estimated and later positions and orientations are estimated by a subsequent algorithm. Direct localization can unfold most of its potential in two cases: Firstly, when the number of antennas is large and massive arrays are employed [56]-[58]. Secondly, when the number of links is large, as in dense cooperative networks [59]. For both cases, it has been shown that direct localization is much more robust with respect to multipath propagation, and thus considerably outperforms the two step approach. With massive arrays and device-to-device (D2D) 
links being considered for $5 \mathrm{G}$ and $6 \mathrm{G}$ cellular networks, direct localization methods receive an increasing amount of attention. The measurements presented in Section VI were done on open grassland, with negligible multipath propagation. Thus the performance of cooperative SLAC in multipath environments is beyond the scope of this paper. However, depending on the envisaged application, multipath propagation could be an issue. Therefore the impact of multipath on cooperative SLAC deserves an investigation.

\section{Sensor Fusion}

This paper focuses exclusively on radio navigation. For a practical system targeting e.g. localization for smart vehicles or robotic swarms, sensor fusion is key to accuracy and reliability. For a robotic platform, proprioceptive sensors like inertial sensors and wheel odometry are typically available. For many applications, it is also desired to sense and map the environment with exteroceptive sensors such as mono or stereo cameras and laser scanners. Such information is also valuable for localization, and is exploited e.g. in SLAM. We propose SLAC within a Bayesian framework, which is well suited for a sensor fusion approach or to be integrated with SLAM. If further sensor are considered, their calibration parameters, e.g. the biases of inertial sensors, can be integrated into the state space. Integrating proprioceptive sensors usually leads to a more accurate prediction. In addition to the improved localization performance, this would be very beneficial for cooperative SLAC, as it could reduce the computation time required for the numerical optimization. With a more accurate prediction, the number of iterations until convergence of the IEKF-like update step (36) could be reduced.

\section{Distributed Estimation}

The SLAC algorithm introduced in Section IV is a centralized estimation scheme. In practice, such an approach could be implemented by sending all measurements from the agents to a fusion center, which perform the calculations and then sends the estimated positions and orientations back to the agents. Depending on the application, a distributed estimation scheme, where each agents performs its own estimations, could be favorable, see [19], [60], [61] and the references therein. In general, by distributed estimation a single point of failure is avoided and the computational load is distributed among the agents. As the aim of this paper is the introduction of cooperative SLAC for radio navigation and a proof-ofconcept with simulations and measurements, a distributed implementation is beyond the scope. Nevertheless, the authors belief this could be an interesting direction for future research.

\section{CONCLUSION}

In this paper we have introduced cooperative SLAC, an approach to calibrate antenna responses and ranging biases simultaneously with localization, without additional sensors. By simulation with random trajectories and antenna responses, we have shown that cooperative SLAC can estimate ranging biases and antenna response deviations. Thus, in the practically relevant case where such biases and deviations exist, the position and orientation RMSE with SLAC has been shown to be more than one order of magnitude lower compared to localization-only. We have further evaluated measurement data from four robotic rovers, where one of them was equipped with a four-port MMA to enable DoA estimation. Analyzing the convergence, we have realized that movement of the agents is crucial for parameter observability. When the calibration parameters have converged, the ranging RMSE calculated over all links in the network was reduced from $0.4 \mathrm{~m}$ to below $0.2 \mathrm{~m}$ with SLAC. Furthermore, the $90^{\text {th }}$ percentile DoA estimation error was reduced from $9.6^{\circ}$ with the antenna response measured in a near-field measurement chamber to $5.9^{\circ}$ with SLAC. We conclude that cooperative SLAC is a viable approach to perform calibration during operation, as it mitigates model mismatch and thus improves position and orientation accuracy.

\section{APPENDIX A}

\section{PARTIAL DERIVATIVES OF LOG-LIKELIHOOD FUNCTION}

First, we calculate the partial derivatives of distance (1) and DoA (2) w.r.t. $\boldsymbol{p}_{i}^{s}$ and $\boldsymbol{p}_{j}^{s}$ :

$$
\begin{gathered}
\frac{\partial d_{i, j}^{s}}{\partial \boldsymbol{p}_{i}^{s}}=\frac{-\left(\boldsymbol{p}_{j}^{s}-\boldsymbol{p}_{i}^{s}\right)}{\left\|\boldsymbol{p}_{j}^{s}-\boldsymbol{p}_{i}^{s}\right\|}=-\frac{\partial d_{i, j}^{s}}{\partial \boldsymbol{p}_{j}^{s}}, \\
\frac{\partial \phi_{i, j}^{s}}{\partial \boldsymbol{p}_{i}^{s}}=\frac{-\left(\boldsymbol{p}_{j}^{s}-\boldsymbol{p}_{i}^{s}\right)}{\left\|\boldsymbol{p}_{j}^{s}-\boldsymbol{p}_{i}^{s}\right\|^{2}}\left[\begin{array}{cc}
0 & 1 \\
-1 & 0
\end{array}\right]=-\frac{\partial \phi_{i, j}^{s}}{\partial \boldsymbol{p}_{j}} .
\end{gathered}
$$

Next, we calculate the partial derivatives of the log-likelihood function $\frac{\partial L_{i, j}^{s}\left(\boldsymbol{x}_{i}^{s}, \boldsymbol{x}_{j}^{s}\right)}{\partial \boldsymbol{x}^{s}}$, which is defined separately for singleport and multiport agents, see (37). For a singleport agent, the partial derivative of (25) w.r.t. ToA $\tau_{i, j}^{s}$ is given by:

$\frac{\partial \tilde{L}_{i, j}^{s}\left(\tau_{i, j}^{s}\right)}{\partial \tau_{i, j}^{s}}=\frac{1}{\sigma_{\boldsymbol{r}_{i, j}^{s}}^{2}} \operatorname{Re}\left\{\frac{2\left(\boldsymbol{r}_{i, j}^{s}\right)^{H}\left(\boldsymbol{s}^{H}\left(\tau_{i, j}^{s}\right) \boldsymbol{r}_{i, j}^{s}\right)}{\left\|\boldsymbol{s}\left(\tau_{i, j}^{s}\right)\right\|^{2}} \frac{\partial \boldsymbol{s}\left(\tau_{i, j}^{s}\right)}{\partial \tau_{i, j}^{s}}\right\}$.

For a multiport agent, the partial derivatives of (27) w.r.t. ToA $\tau_{i, j}^{s}$, DoA $\phi_{i, j}^{s}$ and sampling matrix entries $\boldsymbol{g}_{i}^{s}$ are given by

$$
\begin{aligned}
\frac{\tilde{L}_{i, j}^{s}\left(\phi_{i, j}^{s}, \tau_{i, j}^{s}, \boldsymbol{g}_{i}^{s}\right)}{\partial \phi_{i, j}^{s}}= & \frac{1}{\sigma_{\boldsymbol{r}_{i, j}^{s}}^{2}} \operatorname{Re}\left\{\left(\frac{\partial \tilde{L}_{i, j}^{s}\left(\phi_{i, j}^{s}, \tau_{i, j}^{s}, \boldsymbol{g}_{i}^{s}\right)}{\partial \boldsymbol{e}_{i, j}^{s}}\right)^{H}\right. \\
& \left.\operatorname{vec}\left\{\boldsymbol{G}_{i, j}^{s} \frac{\partial \boldsymbol{b}\left(\phi_{i, j}^{s}\right)}{\partial \phi_{i, j}^{s}} \boldsymbol{s}^{T}\left(\tau_{i, j}^{s}\right)\right\}\right\}, \quad(46) \\
\frac{\tilde{L}_{i, j}^{s}\left(\phi_{i, j}^{s}, \tau_{i, j}^{s}, \boldsymbol{g}_{i}^{s}\right)}{\partial \tau_{i, j}^{s}}= & \frac{1}{\sigma_{\boldsymbol{r}_{i, j}^{s}}^{2}} \operatorname{Re}\left\{\left(\frac{\partial \tilde{L}\left(\phi_{i, j}^{s}, \tau_{i, j}^{s}, \boldsymbol{g}_{i}^{s}\right)}{\partial \boldsymbol{e}_{i, j}^{s}}\right)^{H}\right. \\
& \left(\frac{\partial \boldsymbol{s}\left(\tau_{i, j}^{s}\right)}{\partial \tau_{i, j}^{s}} \otimes\left(\boldsymbol{G}_{i, j}^{s} \boldsymbol{b}\left(\phi_{i, j}^{s}\right)\right)\right\},
\end{aligned}
$$

$$
\begin{aligned}
& \frac{\tilde{L}_{i, j}^{s}\left(\phi_{i, j}^{s}, \tau_{i, j}^{s}, \boldsymbol{g}_{i}^{s}\right)}{\partial \boldsymbol{g}_{i}^{s}}= \\
& {\left[\begin{array}{l}
\operatorname{Re}\left\{\left(\boldsymbol{b}^{*}\left(\phi_{i, j}^{s}\right) \boldsymbol{s}^{H}\left(\tau_{i, j}^{s}\right) \otimes \mathbb{I}_{M_{i}}\right) \frac{\partial \tilde{L}_{i, j}^{s}\left(\phi_{i, j}^{s}, \tau_{i, j}^{s}, \boldsymbol{g}_{i}^{s}\right)}{\partial \boldsymbol{e}_{i, j}^{s}}\right\} \\
\left.\operatorname{Im}\left\{\left(\boldsymbol{b}^{*}\left(\phi_{i, j}^{s}\right) \boldsymbol{s}^{H}\left(\tau_{i, j}^{s}\right) \otimes \mathbb{I}_{M_{i}}\right) \frac{\partial \tilde{L}_{i, j}^{s}\left(\phi_{i, j}^{s}, \tau_{i, j}^{s}, \boldsymbol{g}_{i}^{s}\right)}{\partial \boldsymbol{e}_{i, j}^{s}}\right\}\right],
\end{array}\right.}
\end{aligned}
$$


respectively, with

$$
\begin{aligned}
& \frac{\tilde{L}_{i, j}^{s}\left(\phi_{i, j}^{s}, \tau_{i, j}^{s}, \boldsymbol{g}_{i}^{s}\right)}{\partial \boldsymbol{e}_{i, j}^{s}}= \\
& \quad \frac{-2}{\sigma_{\boldsymbol{r}_{i, j}^{s}}^{2}}\left(\frac{\boldsymbol{e}_{i, j}^{s}\left(\boldsymbol{e}_{i, j}^{s}\right)^{H}}{\left(\boldsymbol{e}_{i, j}^{s}\right)^{H} \boldsymbol{e}_{i, j}^{s}} \boldsymbol{r}_{i, j}^{s}-\boldsymbol{r}_{i, j}^{s}\right)\left(\left(\boldsymbol{r}_{i, j}^{s}\right)^{H} \frac{\boldsymbol{e}_{i, j}^{s}}{\left(\boldsymbol{e}_{i, j}^{s}\right)^{H} \boldsymbol{e}_{i, j}^{s}}\right)
\end{aligned}
$$

We can now compute the partial derivative $\frac{\partial L_{i, j}^{s}\left(\boldsymbol{x}_{i}^{s}, \boldsymbol{x}_{j}^{s}\right)}{\partial \boldsymbol{x}^{s}}$, where only the partial derivatives w.r.t. $\boldsymbol{p}_{i}^{s}, \boldsymbol{p}_{j}^{s}, \psi_{i}^{s}, \delta_{i}^{s}$ and $\boldsymbol{g}_{i}^{s}$ for each node $i \in \mathbb{D}$ are nonzero, see (48) and (50) to (52).

$$
\begin{aligned}
& \frac{\partial L_{i, j}^{s}\left(\boldsymbol{x}_{i}^{s}, \boldsymbol{x}_{j}^{s}\right)}{\partial \boldsymbol{p}_{i}^{s}}=\frac{1}{c} \frac{L_{i, j}^{s}\left(\boldsymbol{x}_{i}^{s}, \boldsymbol{x}_{j}^{s}\right)}{\partial \tau_{i, j}^{s}} \frac{\partial d_{i, j}^{s}}{\partial \boldsymbol{p}_{i}^{s}} \\
&+\frac{L_{i, j}^{s}\left(\boldsymbol{x}_{i}^{s}, \boldsymbol{x}_{j}^{s}\right)}{\partial \phi_{i, j}^{s}} \frac{\partial \phi_{i, j}^{s}}{\partial \boldsymbol{p}_{i}^{s}}=-\frac{\partial L_{i, j}^{s}\left(\boldsymbol{x}_{i}^{s}, \boldsymbol{x}_{j}^{s}\right)}{\partial \boldsymbol{p}_{j}^{s}} \\
& \frac{\partial L_{i, j}^{s}\left(\boldsymbol{x}_{i}^{s}, \boldsymbol{x}_{j}^{s}\right)}{\partial \psi_{i}^{s}}=\frac{L_{i, j}^{s}\left(\boldsymbol{x}_{i}^{s}, \boldsymbol{x}_{j}^{s}\right)}{\partial \phi_{i, j}^{s}} \frac{\partial \phi_{i, j}^{s}}{\partial \psi_{i}^{s}} \\
& \frac{\partial L_{i, j}^{s}\left(\boldsymbol{x}_{i}^{s}, \boldsymbol{x}_{j}^{s}\right)}{\partial \delta_{i}^{s}}=\frac{1}{c} \frac{\partial L_{i, j}^{s}\left(\boldsymbol{x}_{i}^{s}, \boldsymbol{x}_{j}^{s}\right)}{\partial \tau_{i, j}^{s}}
\end{aligned}
$$

\section{REFERENCES}

[1] F. Zafari, A. Gkelias, and K. K. Leung, "A survey of indoor localization systems and technologies," IEEE Commun. Surveys Tuts., vol. 21, no. 3, pp. 2568-2599, 2019

[2] D. Miorandi, S. Sicari, F. De Pellegrini, and I. Chlamtac, "Internet of things: Vision, applications and research challenges," Ad Hoc Networks, vol. 10, no. 7, pp. 1497-1516, Sep. 2012.

[3] P. Figueiredo e Silva, V. Kaseva, and E. S. Lohan, "Wireless positioning in IoT: A look at current and future trends," Sensors, vol. 18, no. 8, Jul. 2018.

[4] S. Kuutti et al., "A survey of the state-of-the-art localization techniques and their potentials for autonomous vehicle applications," IEEE Internet Things J., vol. 5, no. 2, pp. 829-846, Apr. 2018.

[5] A. Fascista, G. Ciccarese, A. Coluccia, and G. Ricci, "Angle of arrivalbased cooperative positioning for smart vehicles," IEEE Trans. Intell. Transp. Syst., vol. 19, no. 9, pp. 2880-2892, Sep. 2018.

[6] O. Kanhere and T. S. Rappaport, "Position location for futuristic cellular communications: $5 \mathrm{G}$ and beyond," IEEE Commun. Mag., vol. 59, no. 1, pp. 70-75, Jan. 2021.

[7] C. De Lima et al., "Convergent communication, sensing and localization in 6G systems: An overview of technologies, opportunities and challenges," IEEE Access, vol. 9, pp. 26902-26925, 2021.

[8] Z. Abu-Shaban, X. Zhou, T. Abhayapala, G. Seco-Granados, and H. Wymeersch, "Error bounds for uplink and downlink 3D localization in 5G millimeter wave systems," IEEE Trans. Wireless Commun., vol. 17, no. 8, pp. 4939-4954, 2018.

[9] A. Shahmansoori, G. E. Garcia, G. Destino, G. Seco-Granados, and H. Wymeersch, "Position and orientation estimation through millimeterwave MIMO in 5G systems," IEEE Trans. Wireless Commun., vol. 17, no. 3, pp. 1822-1835, 2018.

[10] M. J. Schuster et al., "The ARCHES space-analogue demonstration mission: Towards heterogeneous teams of autonomous robots for collaborative scientific sampling in planetary exploration," IEEE Robot. Autom. Lett., pp. 5315-5322, Oct. 2020.

[11] S. Zhang et al., "Self-aware swarm navigation in autonomous exploration missions," Proc. IEEE, vol. 108, no. 7, pp. 1168-1195, Jul. 2020.

[12] A. Guerra, F. Guidi, and D. Dardari, "Single-anchor localization and orientation performance limits using massive arrays: MIMO vs. Beamforming," IEEE Trans. Wireless Commun., vol. 17, no. 8, pp. 5241-5255, Aug. 2018.

[13] R. Pöhlmann et al., "On the potential of multi-mode antennas for direction-of-arrival estimation," IEEE Trans. Antennas Propag., vol. 67, no. 5, pp. 3374-3386, May 2019.
[14] Y. Shen, H. Wymeersch, and M. Z. Win, "Fundamental limits of wideband localization-Part II: Cooperative networks," IEEE Trans. Inf. Theory, vol. 56, no. 10, pp. 4981-5000, Oct. 2010.

[15] Y. Shen and M. Win, "On the accuracy of localization systems using wideband antenna arrays," IEEE Trans. Commun., vol. 58, no. 1, pp 270-280, Jan. 2010.

[16] M. Z. Win, Y. Shen, and W. Dai, "A theoretical foundation of network localization and navigation," Proc. IEEE, vol. 106, no. 7, pp. 1136-1165, Jul. 2018.

[17] J. Schloemann and R. M. Buehrer, "On the value of collaboration in location estimation,” IEEE Trans. Veh. Technol., vol. 65, no. 5, pp. 35853596, May 2016.

[18] A. Conti, M. Guerra, D. Dardari, N. Decarli, and M. Z. Win, "Network experimentation for cooperative localization," IEEE J. Sel. Areas Commun., vol. 30, no. 2, pp. 467-475, Feb. 2012.

[19] R. M. Buehrer, H. Wymeersch, and R. M. Vaghefi, "Collaborative sensor network localization: Algorithms and practical issues," Proc. IEEE, vol. 106, no. 6, pp. 1089-1114, Jun. 2018.

[20] S. R. Zekavat et al., "An overview on position location: Past, present, future," Int. J. Wirel. Inf. Netw., vol. 28, no. 1, pp. 45-76, Mar. 2021.

[21] M. Costa, V. Koivunen, and M. Viberg, "Array processing in the face of nonidealities," in Academic Press Library in Signal Processing, ser. Academic Press Library in Signal Processing, A. M. Zoubir, M. Viberg, R. Chellappa, and S. Theodoridis, Eds. Boston: Elsevier, Jan. 2014, vol. 3, pp. 819-857.

[22] M. J. Schuster et al., "Towards autonomous planetary exploration: The Lightweight Rover Unit (LRU), its success in the SpaceBotCamp challenge, and beyond," J. Intell. \& Robot. Syst., vol. 93, no. 3-4, pp. 461-494, Mar. 2019.

[23] C. Cadena et al., "Past, present, and future of simultaneous localization and mapping: Toward the robust-perception age," IEEE Trans. Robot., vol. 32, no. 6, pp. 1309-1332, Dec. 2016.

[24] E. Foxlin, "Generalized architecture for simultaneous localization, autocalibration, and map-building," in Proc. IEEE/RSJ Int. Conf. Intelligent Robots and System (IROS), vol. 1, Lausanne, Switzerland, 2002, pp. 527-533.

[25] A. Martinelli, N. Tomatis, and R. Siegwart, "Simultaneous localization and odometry self calibration for mobile robot," Autonomous Robots, vol. 22, no. 1, pp. 75-85, Jan. 2007.

[26] H. Miura, T. Yoshida, K. Nakamura, and K. Nakadai, "SLAM-based online calibration for asynchronous microphone array," Advanced Robotics, vol. 26, no. 17, pp. 1941-1965, Dec. 2012.

[27] H. Sadjadi, K. Hashtrudi-Zaad, and G. Fichtinger, "Simultaneous localization and calibration for electromagnetic tracking systems," The Int. J. Med. Robot. Comput. Assist. Surg., vol. 12, no. 2, pp. 189-198, Jun. 2016.

[28] C. Taylor, A. Rahimi, J. Bachrach, H. Shrobe, and A. Grue, "Simultaneous localization, calibration, and tracking in an ad hoc sensor network," in Proc. 5th Int. Conf. Information Processing in Sensor Networks, Nashville, Tennessee, Apr. 2006, pp. 27-33.

[29] N. Petrov, O. Krasnov, and A. G. Yarovoy, "Auto-calibration of automotive radars in operational mode using simultaneous localisation and mapping," IEEE Trans. Veh. Technol., vol. 70, no. 3, pp. 2062-2075, Mar. 2021.

[30] H. Kim et al., "5G mmWave cooperative positioning and mapping using multi-model PHD filter and map fusion," IEEE Trans. Wireless Commun., vol. 19, no. 6, pp. 3782-3795, Jun. 2020.

[31] A. Fascista, A. Coluccia, H. Wymeersch, and G. Seco-Granados, "Downlink single-snapshot localization and mapping with a single-antenna receiver," IEEE Trans. Wireless Commun., vol. 20, no. 7, pp. 46724684, Jul. 2021.

[32] R. Mendrzik, H. Wymeersch, and G. Bauch, "Joint localization and mapping through millimeter wave MIMO in 5G systems," in Proc. IEEE Global Communications Conf. (GLOBECOM), Abu Dhabi, Dec. 2018.

[33] V. N. Ekambaram and K. Ramchandran, "SLAC-RF: Simultaneous 3D localization of mobile readers and calibration of RFID supertags," EECS Department, University of California, Berkeley, Tech. Rep. UCB/EECS2012-188, Aug. 2012.

[34] S. He, S.-H. G. Chan, L. Yu, and N. Liu, "SLAC: Calibration-free pedometer-fingerprint fusion for indoor localization," IEEE Trans. Mobile Comput., vol. 17, no. 5, pp. 1176-1189, May 2018.

[35] E. Staudinger, S. Zhang, R. Pöhlmann, and A. Dammann, "The role of time in a robotic swarm: A joint view on communications, localization, and sensing," IEEE Commun. Mag., vol. 59, no. 2, pp. 98-104, Feb. 2021.

[36] C. A. Balanis and P. I. Ioannides, Introduction to Smart Antennas. San Rafael: Morgan \& Claypool Publishers, 2007. 
[37] M. Viberg and A. Zoubir, Array and Statistical Signal Processing, ser. Academic Press Library in Signal Processing. Boston: Academic Press, 2014.

[38] M. A. Doron and E. Doron, "Wavefield modeling and array processing. I. Spatial sampling," IEEE Trans. Signal Process., vol. 42, no. 10, pp. 2549-2559, Oct. 1994.

[39] M. Costa, A. Richter, and V. Koivunen, "Unified array manifold decomposition based on spherical harmonics and 2-D fourier basis," IEEE Trans. Signal Process., vol. 58, no. 9, pp. 4634-4645, Sep. 2010.

[40] X. Rong Li and V. Jilkov, "Survey of maneuvering target tracking. Part I: Dynamic models," IEEE Trans. Aerosp. Electron. Syst., vol. 39, no. 4, pp. 1333-1364, Oct. 2003.

[41] M. T. Busch and S. S. Blackman, "Evaluation of IMM filtering for an air defense system application," in Proc. SPIE's 1995 Int. Symp. Optical Science, Engineering, and Instrumentation, O. E. Drummond, Ed., San Diego, CA, Sep. 1995, pp. 435-447.

[42] S. Thrun, W. Burgard, and D. Fox, Probabilistic Robotics. MIT Press, Aug. 2005.

[43] D. Pauluzzi and N. Beaulieu, "A comparison of SNR estimation techniques for the AWGN channel," IEEE Trans. Commun., vol. 48, no. 10, pp. 1681-1691, Oct. 2000.

[44] M. Wax and A. Leshem, "Joint estimation of time delays and directions of arrival of multiple reflections of a known signal," IEEE Trans. Signal Process., vol. 45, no. 10, pp. 2477-2484, Oct. 1997.

[45] Y. Bar-Shalom, X. R. Li, and T. Kirubarajan, Estimation with Applications to Tracking and Navigation: Theory Algorithms and Software. John Wiley \& Sons, 2004.

[46] R. Fletcher, Practical Methods of Optimization. Chichester, UK: Wiley, 1987.

[47] L. Tierney and J. B. Kadane, "Accurate approximations for posterior moments and marginal densities," J. Amer. Statist. Assoc., vol. 81, no. 393, pp. 82-86, Mar. 1986

[48] H. Naseri and V. Koivunen, "A Bayesian algorithm for distributed cooperative localization using distance and direction estimates," IEEE Trans. Signal Inf. Process. Netw., pp. 290-304, Nov. 2018.

[49] B. Q. Ferreira, J. Gomes, C. Soares, and J. P. Costeira, "FLORIS and CLORIS: Hybrid source and network localization based on ranges and video," Signal Processing, vol. 153, pp. 355-367, Dec. 2018.

[50] S. Tomic, M. Beko, and R. Dinis, "3-D target localization in wireless sensor networks using RSS and AoA measurements," IEEE Trans. Veh. Technol., vol. 66, no. 4, pp. 3197-3210, Apr. 2017.

[51] S. Caizzone, M. S. Circiu, W. Elmarissi, and C. Enneking, "All-GNSSband DRA antenna for high-precision applications," in Proc. 12th European Conf. Antennas and Propagation (EuCAP), Apr. 2018, pp. 543-547.

[52] R. Pöhlmann, G. Pedregosa, S. Caizzone, E. Staudinger, and P. A. Hoeher, "Multi-mode antenna enabled direction-of-arrival estimation for swarm navigation," in Proc. 16th Workshop Positioning, Navigation and Communications (WPNC), Bremen, Germany, Oct. 2019.

[53] ANavS GmbH, "ANavS Multi-Sensor RTK Module," https://www.anavs.de.

[54] S. Zhang, R. Pöhlmann, E. Staudinger, and A. Dammann, "Assembling a swarm navigation system: Communication, localization, sensing and control," in Proc. 1st IEEE Int. Workshop Communication and Networking for Swarms Robotics (RoboCom), Online, Jan. 2021.

[55] P. Closas and A. Gusi-Amigo, "Direct position estimation of GNSS receivers: Analyzing main results, architectures, enhancements, and challenges," IEEE Signal Process. Mag., vol. 34, no. 5, pp. 72-84, Sep. 2017.

[56] H. Zhao, N. Zhang, and Y. Shen, "Beamspace direct localization for large-scale antenna array systems," IEEE Trans. Signal Process., pp. 3529-3544, May 2020.

[57] A. Fascista, A. Coluccia, and G. Ricci, "A pseudo maximum likelihood approach to position estimation in dynamic multipath environments," Signal Processing, vol. 181, p. 107907, Apr. 2021.

[58] N. Garcia, H. Wymeersch, E. G. Larsson, A. M. Haimovich, and M. Coulon, "Direct localization for massive MIMO," IEEE Trans. Signal Process., vol. 65, no. 10, pp. 2475-2487, May 2017.

[59] S. Zhang et al., "Distributed direct localization suitable for dense networks," IEEE Trans. Aerosp. Electron. Syst., pp. 1209-1227, Apr. 2020.

[60] S. Safavi, U. A. Khan, S. Kar, and J. M. F. Moura, "Distributed localization: A linear theory," Proc. IEEE, pp. 1204-1223, 2018.

[61] A. Ihler, J. Fisher, R. Moses, and A. Willsky, "Nonparametric belief propagation for self-localization of sensor networks," IEEE J. Sel. Areas Commun., vol. 23, no. 4, pp. 809-819, Apr. 2005.

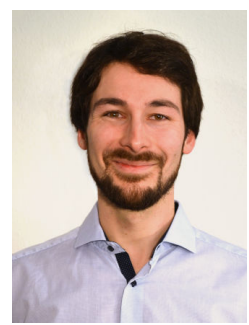

Robert Pöhlmann received the B.Sc. and the M.Sc. degrees in Electrical Engineering and Information Technology from the Technical University of $\mathrm{Mu}$ nich (TUM) in 2014 and 2016, respectively. In 2013 he joined the Institute of Communications and Navigation of the German Aerospace Center (DLR) as a student trainee and in 2016 he became a research staff member. His current research interests are in the area of statistical signal processing for multiport antennas and cooperative localization.

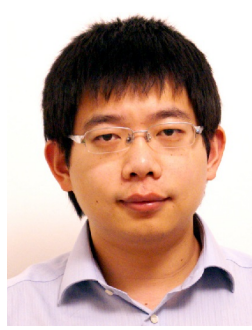

Siwei Zhang received the B.Sc. in electrical engineering from the Zhejiang University, China, in 2009, the M.Sc. in communication engineering from the Technical University of Munich, Germany, in 2011, and the Dr.-Ing. (Ph.D.) in electrical engineering from the University of Kiel, Germany, in 2020. In 2012, he joined the Institute of Communications and Navigation, German Aerospace Center (DLR), Germany, as a Research Staff Member. His research interests include radio navigation, cooperative positioning and swarm navigation.

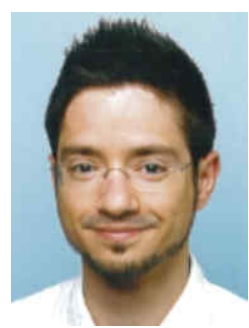

Emanuel Staudinger received an M.Sc. in embedded systems design from the University of Applied Sciences of Hagenberg, Austria, in 2010, when he joined the Institute of Communications and Navigation of the German Aerospace Center (DLR), Munich, Germany, as a research staff member. He received a Ph.D. with distinction from the Institute of Electrodynamics and Microelectronics at the University of Bremen, Germany, in 2015. His current research interests include system design for cooperative positioning, experimental platform design based on SDRs, and experimental validation for swarm navigation.

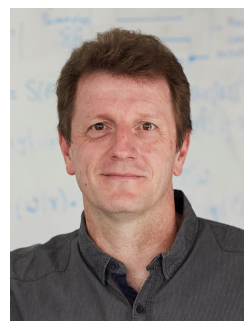

Armin Dammann received the Dipl.-Ing. (M.Sc.) and Dr.-Ing. (Ph.D.) degrees in Electrical Engineering from the University of Ulm, Germany, in 1997 and 2005 respectively. In 1997 he joined the Institute of Communications and Navigation of the German Aerospace Center (DLR) as a research staff member. Since 2005 he is head of the Mobile Radio Transmission Research Group. His research interest and activities include signal design and signal processing for terrestrial wireless communication and navigation systems. In these fields, he has been active in several EU-projects, e.g., WINNER, WHERE and WHERE2. Armin Dammann is lecturer at the Technical University of Munich for "Robot and Swarm Navigation".

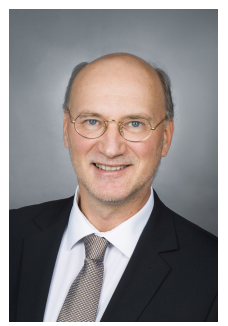

Peter A. Hoeher (F'14) received the Dipl.Ing. (M.Sc.) degree in electrical engineering from RWTH Aachen University, Aachen, Germany, in 1986, and the Dr.Ing. (Ph.D.) degree in electrical engineering from the University of Kaiserslautern, Kaiserslautern, Germany, in 1990. From 1986 to 1998 , he was with the German Aerospace Center (DLR), Oberpfaffenhofen, Germany. From 1991 to 1992, he was on leave at AT\&T Bell Laboratories, Murray Hill, NJ. Since 1998 he is a Full Professor of electrical and information engineering at Kiel University, Kiel, Germany. His research interests are in the general area of wireless communications and applied information theory. Since 2014, he has been a Fellow of the IEEE for contributions to decoding and detection that include reliability information. 